\title{
Development of gender- and age group-specific equations for estimating body weight from anthropometric measurement in Thai adults
}

This article was published in the following Dove Press journal:

International Journal of General Medicine

19 January 2012

Number of times this article has been viewed

\section{Kaweesak \\ Chittawatanarat ${ }^{1,2}$ \\ Sakda Pruenglampoo 3 \\ Vibul Trakulhoon ${ }^{4}$ \\ Winai Ungpinitpong 5 \\ Jayanton Patumanond ${ }^{2}$ \\ 'Department of Surgery, Faculty of Medicine, ${ }^{2}$ Clinical Epidemiology Unit, ${ }^{3}$ Research Institute for Health Sciences, Chiang Mai University, Chiang Mai, Thailand; ${ }^{4}$ Department of Surgery, Bhumibol Adulyadej Hospital, Bangkok, Thailand; ${ }^{5}$ Surgical Unit, Surin Hospital, Surin, Thailand}

Video abstract

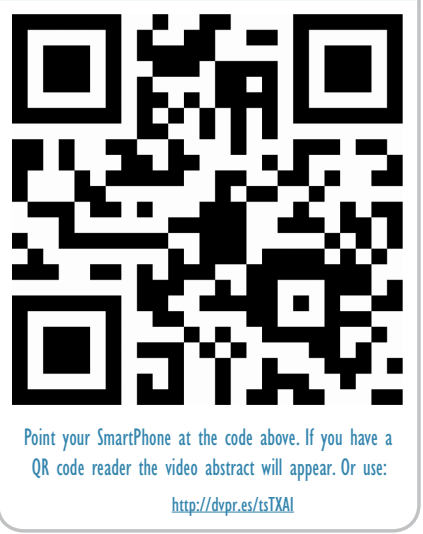

Correspondence: Kaweesak Chittawatanarat

Department of Surgery, Faculty of Medicine, Maharaj Nakorn Chiang Mai Hospital, Chiang Mai University, Amphur Muang, Chiang Mai, Thailand 50200

Tel +6653945 533

Fax +66 53946 I39

Email kchittaw@gmail.com
Background: Many medical procedures routinely use body weight as a parameter for calculation. However, these measurements are not always available. In addition, the commonly used visual estimation has had high error rates. Therefore, the aim of this study was to develop a predictive equation for body weight using body circumferences.

Methods: A prospective study was performed in healthy volunteers. Body weight, height, and eight circumferential level parameters including neck, arm, chest, waist, umbilical level, hip, thigh, and calf were recorded. Linear regression equations were developed in a modeling sample group divided by sex and age (younger $<60$ years and older $\geq 60$ years). Original regression equations were modified to simple equations by coefficients and intercepts adjustment. These equations were tested in an independent validation sample.

Results: A total of 2000 volunteers were included in this study. These were randomly separated into two groups (1000 in each modeling and validation group). Equations using height and one covariate circumference were developed. After the covariate selection processes, covariate circumference of chest, waist, umbilical level, and hip were selected for single covariate equations (Sco). To reduce the body somatotype difference, the combination covariate circumferences were created by summation between the chest and one torso circumference of waist, umbilical level, or hip and used in the equation development as a combination covariate equation (Cco). Of these equations, Cco had significantly higher $10 \%$ threshold error tolerance compared with Sco (mean percentage error tolerance of Cco versus Sco [95\% confidence interval; 95\% CI]: 76.9 [74.2-79.6] versus 70.3 [68.4-72.3]; $P<0.01$, respectively). Although simple covariate equations had more evidence errors than the original covariate equations, there was comparable error tolerance between the types of equations (original versus simple: 74.5 [71.9-77.1] versus 71.7 [69.2-74.3]; $P=0.12$, respectively). The chest containing covariate $(\mathrm{C})$ equation had the most appropriate performance for Sco equations (chest versus nonchest: 73.4 [69.7-77.1] versus 69.3 [67.0-71.6]; $P=0.03$, respectively). For Cco equations, although there were no differences between covariates using summation of chest and hip $(\mathrm{C}+\mathrm{Hp})$ and other $\mathrm{Cco}$ but $\mathrm{C}+\mathrm{Hp}$ had a slightly higher performance validity $(\mathrm{C}+\mathrm{Hp}$ versus other Cco [95\% CI]: 77.8 [73.2-82.3] versus 76.5 [72.7-80.2]; $P=0.65$, respectively).

Conclusion: Body weight can be predicted by height and circumferential covariate equations. Cco had more Sco error tolerance. Original and simple equations had comparable validity. Chest- and $\mathrm{C}+\mathrm{Hp}$-containing covariate equations had more precision within the Sco and Cco equation types, respectively.

Keywords: body weight, anthropometry, circumference, Thai, linear models

\section{Introduction}

One of the common important clinical measurement parameters is body weight. Many clinical situations utilize body weight as a variable for the determination of nutrition 
requirements, drug dose administration, resuscitation process, pulmonary tidal volume estimation and hemodynamic assessments. ${ }^{1-4}$ However, there are many limitations to obtaining body weight in some clinical practice situations especially in nonambulatory elderly people, and emergency and critically ill patients. A special instrument is required for direct measurement in these patients. Nevertheless, it might be unavailable due to limited resources in developing countries. Although visual estimation is the most common method of estimating weight, the current literature has reported great inaccuracies with this method compared with the actual body weight. In addition, the precision of this method is operatordependent. ${ }^{5-8}$ These errors might lead to adverse and ineffective treatment outcomes. ${ }^{8,9}$ To diminish predictive error, one study that was performed in an emergency department (ED) setting demonstrated that anthropometric measurement had greater accuracy of around $20 \%$ within a $10 \%$ error threshold than visual estimation by ED providers. ${ }^{10}$ Although these more scientific anthropometric measurements to estimate body weight have been proposed, ethnic differences and measurement parameter distinctions might impact predicted validity. ${ }^{11-14}$ In addition, some parameters used in equations are hard to assess in general practice especially those requiring skinfold thickness. ${ }^{10,15,16}$ To our best knowledge, there is no recommended formula to predict body weight with circumferential anthropometric parameters in the Thai or Asian populations. Therefore, the aims of this study were to obtain appropriate and precise methods to estimate actual body weight using circumferential parameters from different parts of the body as well as to propose a simple estimation equation with acceptable validity which could be applied conveniently for general medical practice.

\section{Methods}

The authors performed a prospective cross-sectional study which enrolled healthy Thai adult volunteers by an invitation announcement to the Faculty of Medicine, Chiang Mai University via public information posters and the hospital Web site. Four research assistants were trained in the measurement method for each circumferential anthropometric parameter and reliability testing was performed before data collection with kappa agreement with more than $95 \%$ with up to $5 \%$ error. The authors excluded volunteers whose age was less than 18 years, amputated $\operatorname{limb}(\mathrm{s})$, inability of ambulation, inability to lie down, and chronic disease which might interfere with measured parameters such as liver cirrhosis, renal failure, chronic steroid use, and edematous limb(s). This study was approved by the Faculty of Medicine, Chiang Mai University Ethics Committee.

The authors measured and collected body weight and body circumferences as well as demographic data, sex, age, occupation, and habitats. Body circumferences were measured in supine position with a cloth tape measure up to $1 \mathrm{~mm}$ width at eight levels including neck, chest, waist, umbilical level, and hip, arm, thigh, and leg circumferences. The measurement method and reference points are described in Table 1. Actual body weight was measured by the same digital weighing apparatus (Zepper TCA-200A-RT; Bangkok, Thailand) and recorded in kilograms with one decimal point. Height was measured by a standard measurement board and all subjects were positioned for height measurement with head, shoulder blades, buttocks, and heels touching the board. This measurement was recorded in centimeters.

The study sample was separated randomly and independently into two groups, a regression modeling group, in whom regression equations were developed to estimate body weight, and a validation group, in whom the equations were tested. The estimated sample size in each group was 250 volunteers based on differences of physiological status and body composition between younger and elderly people as well as each gender distinction. ${ }^{17}$ The authors further divided the people by age group and sex. Age was classified into two

Table I Methods of anthropometric circumferential parameter measurements

\begin{tabular}{ll}
\hline Covariates & Point of measurement method \\
\hline Neck & $\begin{array}{l}\text { Level at cricoid cartilage in anterior and midpoint between external occipital protuberance and tip of spinous process } \\
\text { of } 7 \text { th cervical spine (vertebral prominens at root of neck) in posterior } \\
\text { Level at midpoint between tip of acromioclavicular eminent to tip of olecranon of elbow of nondominant arm }\end{array}$ \\
Arm & $\begin{array}{l}\text { At full expiration, measurement at upper chest on the level of junction between the deltopectoral groove and tip } \\
\text { of anterior axillary fold }\end{array}$ \\
Waist & Narrowest part of abdominal circumference above umbilicus or measurement above umbilicus I-I.5 inches in cases \\
that could not identify the narrowest part & Level of umbilicus at anterior and about I.5-2.0 inches above the superior posterior iliac spine at posterior \\
Hip & Widest part of hip, level of pubic symphysis at anterior and ischial tuberosity at posterior \\
Thigh & Level at midpoint between inguinal point and upper border of patella \\
Calf & Level at midpoint between heel and upper most point of femur condyles (approximately 4 cm proximal to the patella)
\end{tabular}


groups with a cut-off at 60 years by the official retirement age in the authors' country as well as a previous study background in which there were different body compositions in elderly people. ${ }^{17}$ The total estimated population included in this study was 2000 healthy volunteers.

\section{Statistical analysis, parameters selection, modeling, and validation}

All of the continuous variable data between age groups and sex were tested for normal distribution with a visual inspection of the histogram and the Shapiro-Wilk W test and reported as mean \pm SD. Group differences were calculated using Student's $t$-test for normally distributed continuous variables and Mann-Whitney $U$ test for nonparametric continuous variables. The univariable and multivariable linear regression model was used to identify the relationship between independent variable(s) and body weight. A statistical difference was defined as $P$ value less than 0.05 .

Covariate parameters were decided for the modeling selection by considering correlation values between circumferential variables and body weight. For the equation creation, the authors conformed to the basic theoretical background of alteration of weight depending on the height and volume of an object. Therefore, height was included in the equation covariate in all of the calculated formulas. ${ }^{11}$ The authors developed an estimation equation for body weight divided by age group and sex. The formula used was as follows:

$$
\text { Body weight }(\mathrm{kg})=b_{1}(\text { Covariate })+b_{2}(\text { Height })+a
$$

where $\left(b_{1}\right)$ and $\left(b_{2}\right)$ were the regression coefficients and $(a)$ represented the intercept.

Equations using these single circumferential variables for prediction were determined as single covariate equation or formula (Sco). The authors had concerns that individual disproportion of the body figure in chest and torso might affect the model validation and might result in prediction error. Therefore, the combination of circumference of chest together with hip, waist or umbilical level circumference were performed (Chest + Hip $[\mathrm{C}+\mathrm{Hp}]$; Chest + Umbilical level $[\mathrm{C}+\mathrm{U}]$ and Chest + Waist, $[\mathrm{C}+\mathrm{W}])$ and behaved as an independent covariate in the present study equations. These summation-containing variables were determined as combination covariates equation or formula (Cco). The model structure of linearity or violation of linearity between covariates and body weight were verified by residuals versus fitting and predictor plots. To provide the simplest formula, numbers of entered covariates were limited as much as possible in each regression model. Forward and backward stepwise regressions were performed. Multicollinearity covariates in the regression model were separated into independent models. Individual models were selected for further validation based on comparison of adjusted R-square value, Akaike's information criteria (AIC) and Bayesian's information criteria (BIC) in each model prediction. The original regression formulas were modified to simple formulas with adjusted covariate coefficients and constant value to ordinary and memorized number. First, covariate coefficient values were estimated and titrated to the nearest value which could accompany the same value between gender and age group in each covariate equation. Second, mean covariate values were substituted and an intercept value was estimated to the nearest number in each equation. In the case of difference error after modified formula, the coefficient would be adjusted and titrated to minimized error. The final adjusted coefficients and intercept was defined as the modified simple formula.

For external validation, predicted body weight was calculated and the difference was compared to the actual body weight in the other equal-sized volunteer in each validation subgroup. The deviated value was reported in error quantity and relative error to actual body weight in percent. Original regression formulas (original formula) and modified simple formulas (simple formula) were compared together with correlation coefficient, error quantity, and relative error. Absolute errors (predicted weight - actual weight) were compared between equations and stratified by gender and equation types (original or simple formulas) using the paired $t$-test. The performances of equations between type equations were tested by level of relative error which was divided into two groups with error more than $10 \%$ or $20 \%$ of actual body weight. These cut-points were based on previous studies. ${ }^{10,11}$ The agreements of two methods were tested by kappa statistics based on the relative error level. In addition, percentage of error tolerance $(100$ - percentage of error) in the $10 \%$ and $20 \%$ thresholds were reported in each covariate equations.

\section{Results}

From May 2010 through May 2011, 2000 volunteers were included in this study and divided into four subgroups as mentioned previously. In Table 2, there were no differences between the modeling and validation group of all collected variables. However, almost all volunteers $(96.0 \%$ to $99.6 \%$ ) were registered residents in the northern region of Thailand. 
Table 2 Characteristics of subjects in model formulation and validation group classified by sex and age groups

\begin{tabular}{|c|c|c|c|c|c|c|}
\hline \multirow[t]{2}{*}{ Parameters } & \multicolumn{2}{|c|}{ Age $<60$ years } & \multirow[t]{2}{*}{$P$} & \multicolumn{2}{|c|}{ Age $\geq 60$ years } & \multirow[t]{2}{*}{$P$} \\
\hline & Modeling & Validation & & Modeling & Validation & \\
\hline \multicolumn{7}{|l|}{ Sex (\%) } \\
\hline Female & $250(50)$ & $250(50)$ & 1.00 & $250(50)$ & $250(50)$ & 1.00 \\
\hline Male & $250(50)$ & $250(50)$ & & $250(50)$ & $250(50)$ & \\
\hline \multicolumn{7}{|l|}{ Profession (\%) } \\
\hline \multicolumn{7}{|l|}{ Female } \\
\hline Farmer & $36(14.40)$ & $47(18.80)$ & 0.50 & $32(12.80)$ & $31(12.40)$ & 0.59 \\
\hline Officer & $37(14.80)$ & $39(15.60)$ & & $36(14.40)$ & $44(17.60)$ & \\
\hline Private & $33(13.20)$ & $39(15.60)$ & & $15(6.00)$ & $20(8.00)$ & \\
\hline Worker & $79(31.60)$ & $69(27.60)$ & & $18(7.20)$ & $22(8.80)$ & \\
\hline Others & $65(26.00)$ & $56(22.40)$ & & $149(59.60)$ & $133(53.20)$ & \\
\hline \multicolumn{7}{|l|}{ Male } \\
\hline Farmer & $4 \mid(16.53)$ & $43(17.34)$ & 0.83 & $50(20.00)$ & $37(14.80)$ & 0.37 \\
\hline Officer & $43(17.34)$ & 47 (I8.95) & & $40(16.00)$ & $39(15.60)$ & \\
\hline Private & $35(14.11)$ & 28 (II.29) & & $17(6.80)$ & $17(6.80)$ & \\
\hline Worker & $82(33.06)$ & $88(35.48)$ & & $28(11.20)$ & $22(8.80)$ & \\
\hline Others & 47 (18.95) & $42(16.94)$ & & $115(46.00)$ & $135(54.00)$ & \\
\hline \multicolumn{7}{|l|}{ Habitats (\%) } \\
\hline \multicolumn{7}{|l|}{ Female } \\
\hline Northern & $240(96.0)$ & $242(96.8)$ & 0.63 & $247(98.80)$ & $246(98.40)$ & 0.70 \\
\hline Others & $10(4.0)$ & $8(3.2)$ & & $3(1.20)$ & $4(1.60)$ & \\
\hline \multicolumn{7}{|l|}{ Male } \\
\hline Northern & 244 (98.39) & $248(99.60)$ & 0.18 & $248(99.20)$ & $248(99.20)$ & 1.00 \\
\hline Other & $4(1.6 I)$ & I $(0.40)$ & & $2(0.80)$ & $2(0.80)$ & \\
\hline \multicolumn{7}{|l|}{ Age (years) } \\
\hline Female & $46.34 \pm 10.21$ & $45.80 \pm 9.87$ & $0.6 I$ & $67.22 \pm 6.24$ & $67.43 \pm 6.71$ & 0.72 \\
\hline Male & $43.63 \pm 11.28$ & $43.85 \pm 11.10$ & 0.83 & $69.22 \pm 7.41$ & $69.75 \pm 7.60$ & 0.43 \\
\hline \multicolumn{7}{|l|}{ Body weight $(\mathrm{kg})$} \\
\hline Female & $57.89 \pm 10.41$ & $57.06 \pm 10.50$ & 0.38 & $54.27 \pm 10.27$ & $54.51 \pm 10.73$ & 0.80 \\
\hline Male & $67.55 \pm 10.85$ & $67.66 \pm 11.52$ & 0.91 & $60.62 \pm 10.84$ & $59.84 \pm 11.75$ & 0.44 \\
\hline \multicolumn{7}{|l|}{ Height (cm) } \\
\hline Female & $155.62 \pm 5.46$ & $|54.94 \pm 5.6|$ & 0.17 & $152.13 \pm 6.60$ & $|5| .48 \pm 6.43$ & 0.26 \\
\hline Male & $166.42 \pm 6.29$ & $166.09 \pm 6.36$ & 0.55 & $162.65 \pm 7.11$ & $161.72 \pm 7.02$ & 0.14 \\
\hline BMI $\left(\mathrm{kg} / \mathrm{m}^{2}\right)$ & & & & & & \\
\hline Female & $23.74 \pm 4.00$ & $23.89 \pm 3.95$ & 0.67 & $23.39 \pm 3.90$ & $23.69 \pm 4.10$ & 0.41 \\
\hline Male & $24.37 \pm 3.55$ & $24.50 \pm 3.82$ & 0.68 & $22.84 \pm 3.36$ & $22.78 \pm 3.65$ & 0.85 \\
\hline Neck (cm) & & & & & & \\
\hline Female & $33.80 \pm 2.82$ & $33.54 \pm 2.89$ & 0.31 & $33.82 \pm 2.96$ & $33.95 \pm 2.89$ & 0.60 \\
\hline Male & $38.50 \pm 2.94$ & $38.77 \pm 3.09$ & 0.32 & $37.53 \pm 3.03$ & $37.52 \pm 3.36$ & 0.99 \\
\hline Chest (cm) & & & & & & \\
\hline Female & $86.74 \pm 8.25$ & $86.20 \pm 8.05$ & 0.46 & $87.47 \pm 8.33$ & $87.82 \pm 8.67$ & 0.64 \\
\hline Male & $92.61 \pm 7.20$ & $92.72 \pm 7.62$ & 0.87 & $90.34 \pm 7.20$ & $89.82 \pm 7.94$ & 0.45 \\
\hline Hip (cm) & & & & & & \\
\hline Female & $94.32 \pm 8.26$ & $93.56 \pm 8.4 I$ & 0.31 & $94.92 \pm 9.22$ & $95.36 \pm 9.41$ & 0.60 \\
\hline Male & $95.44 \pm 7.51$ & $95.26 \pm 7.95$ & 0.79 & $94.76 \pm 8.06$ & $94.05 \pm 8.68$ & 0.35 \\
\hline Umbilical (cm) & & & & & & \\
\hline Female & $81.37 \pm 10.16$ & $80.47 \pm 10.09$ & 0.32 & $84.30 \pm 10.98$ & $84.89 \pm 10.97$ & 0.55 \\
\hline Male & $84.69 \pm 9.64$ & $84.76 \pm 9.69$ & 0.93 & $86.33 \pm 9.23$ & $85.44 \pm 9.73$ & 0.29 \\
\hline $\operatorname{Arm}(\mathrm{cm})$ & & & & & & \\
\hline Female & $28.13 \pm 3.47$ & $27.81 \pm 3.34$ & 0.29 & $27.40 \pm 3.55$ & $27.57 \pm 3.18$ & 0.56 \\
\hline Male & $29.84 \pm 3.11$ & $29.86 \pm 3.21$ & 0.93 & $28.28 \pm 2.97$ & $27.90 \pm 3.20$ & 0.18 \\
\hline Waist (cm) & & & & & & \\
\hline Female & $77.54 \pm 9.65$ & $76.64 \pm 9.89$ & 0.31 & $80.64 \pm 9.93$ & $81.26 \pm 10.16$ & 0.49 \\
\hline Male & $82.25 \pm 9.30$ & $82.56 \pm 9.57$ & 0.71 & $83.49 \pm 8.74$ & $82.76 \pm 9.31$ & 0.37 \\
\hline Thigh (cm) & & & & & & \\
\hline Female & $46.99 \pm 5.64$ & $46.64 \pm 5.50$ & 0.48 & $42.94 \pm 5.92$ & $43.53 \pm 5.96$ & 0.27 \\
\hline Male & $47.85 \pm 5.47$ & $47.64 \pm 5.94$ & 0.69 & $42.85 \pm 5.01$ & $42.65 \pm 5.56$ & 0.67 \\
\hline Calf (cm) & & & & & & \\
\hline Female & $33.46 \pm 3.51$ & $33.40 \pm 3.55$ & 0.86 & $32.5 \mathrm{I} \pm 4.37$ & $32.32 \pm 4.15$ & 0.61 \\
\hline Male & $35.16 \pm 4.54$ & $35.07 \pm 4.7 \mid$ & 0.82 & $33.37 \pm 4.54$ & $33.14 \pm 4.60$ & 0.58 \\
\hline
\end{tabular}

Abbreviation: BMI, body mass index. 
At the variables selection process after forward and backward stepwise linear regression, the authors found that the torso circumferences of waist, hip, and umbilical level had multicollinearity properties with each other in the modelcreating covariates and these were the major reason to enter these variables separately in each model.

In Table 3, although there were significant correlations of all circumference parameters, only chest, hip, umbilical level, waist, arm, and thigh circumference had a correlation coefficient of more than $70 \%$ in at least three quarters of all subgroups in each covariate equation. However, the authors selected only chest, hip, umbilical level, and waist circumference for further validation and performance assessments after consideration of R-square, $\mathrm{AIC}$, and $\mathrm{BIC}$ values (Table 3). Cco equations of $\mathrm{C}+\mathrm{Hp}, \mathrm{C}+\mathrm{U}$, and $\mathrm{C}+\mathrm{W}$ showed increased correlation coefficients and $\mathrm{R}$-square value as well as decreases in the AIC and BIC values when they were compared to the same level of the Sco (Table 3). Therefore, the authors finally decided to select equations comprised of chest, hip, umbilicus, and waist, $\mathrm{C}+\mathrm{Hp}, \mathrm{C}+\mathrm{U}$, and $\mathrm{C}+\mathrm{W}$ to validate the processes (Table 4).

The coefficients of the equation were confined to a simple number and the intercept of the equation was also adjusted using the average of the covariates values. These modified simple formulas were demonstrated in Table 4. Although correlation coefficients were lower in some simple formulas, most of them were comparable and all had a statistically significant relation with a $P$ value of less than 0.01 (Table 5). While the simple equations of weight prediction could be switched between sex in elderly volunteers except waist-containing covariate equations (Waist and $\mathrm{C}+\mathrm{W}$ equations) in younger volunteers, only

Table 3 Correlation coefficient ( $r$ ), adjusted R-square $\left(R^{2}\right)$, Akaike's information criteria (AIC), and Bayesian's information (BIC) of single and combination covariates classified by sex and age groups

\begin{tabular}{|c|c|c|c|c|c|c|c|c|}
\hline \multirow[t]{2}{*}{ Covariate } & \multicolumn{4}{|c|}{$<60$ years } & \multicolumn{4}{|c|}{$\geq 60$ years } \\
\hline & $r$ & $R^{2}$ & AIC & BIC & $r$ & $R^{2}$ & AIC & BIC \\
\hline \multicolumn{9}{|l|}{ Neck } \\
\hline Female & 0.70 & 0.54 & 1689.00 & 1699.56 & 0.69 & 0.58 & 1660.84 & $|67| .4 \mid$ \\
\hline Male & 0.65 & 0.55 & 1704.94 & $17 \mid 5.50$ & 0.72 & 0.64 & 1649.64 & 1660.20 \\
\hline \multicolumn{9}{|l|}{ Chest } \\
\hline Female & 0.84 & 0.75 & 1535.90 & I546.46 & 0.81 & 0.73 & I553.37 & 1563.94 \\
\hline Male & 0.80 & 0.69 & 1615.30 & 1625.86 & 0.81 & 0.70 & 1605.35 & 1615.92 \\
\hline \multicolumn{9}{|l|}{ Hip } \\
\hline Female & 0.84 & 0.72 & 1560.60 & $157 \mid .17$ & 0.76 & 0.67 & 1602.66 & 1613.22 \\
\hline Male & 0.82 & 0.71 & 1592.28 & 1602.84 & 0.74 & 0.62 & 1661.14 & 1671.70 \\
\hline \multicolumn{9}{|l|}{ Umbilical } \\
\hline Female & 0.84 & 0.78 & 1509.53 & 1520.09 & 0.66 & 0.62 & 1636.88 & 1647.44 \\
\hline Male & 0.81 & 0.73 & 1578.45 & 1589.02 & 0.78 & 0.72 & I587.35 & I597.9| \\
\hline \multicolumn{9}{|l|}{ Arm } \\
\hline Female & 0.77 & 0.69 & |594.|I & 1604.68 & 0.78 & 0.68 & $|589.0|$ & 1599.58 \\
\hline Male & 0.70 & 0.58 & 1687.81 & 1698.38 & 0.71 & 0.62 & 1660.59 & 1671.15 \\
\hline \multicolumn{9}{|l|}{ Waist } \\
\hline Female & 0.87 & 0.82 & 1457.49 & | 468.05 & 0.68 & 0.62 & 1635.62 & 1646.19 \\
\hline Male & 0.82 & 0.75 & 1560.49 & $|57| .05$ & 0.79 & 0.73 & I573.42 & 1583.98 \\
\hline \multicolumn{9}{|l|}{ Thigh } \\
\hline Female & 0.74 & 0.59 & 1661.03 & $|67| .60$ & 0.74 & 0.62 & 1637.98 & 1648.54 \\
\hline Male & 0.61 & 0.48 & 1741.29 & $|75| .86$ & 0.80 & 0.69 & 1613.92 & 1624.49 \\
\hline \multicolumn{9}{|l|}{ Calf } \\
\hline Female & 0.61 & 0.44 & 1739.78 & 1750.3 & 0.68 & 0.54 & 1680.49 & 1691.05 \\
\hline Male & 0.53 & 0.40 & 1776.26 & 1786.82 & 0.64 & 0.55 & 1704.52 & 1715.08 \\
\hline \multicolumn{9}{|l|}{$\mathrm{C}+\mathrm{Hp}$} \\
\hline Female & 0.90 & 0.83 & 1438.54 & |449.| | & 0.83 & 0.76 & $|52| .05$ & 1531.62 \\
\hline Male & 0.88 & 0.79 & 1509.10 & 1519.67 & 0.84 & 0.74 & 1569.06 & 1579.62 \\
\hline \multicolumn{9}{|l|}{$\mathrm{C}+\mathrm{U}$} \\
\hline Female & 0.88 & 0.82 & $|45| .23$ & $|46| .79$ & 0.78 & 0.72 & I555.27 & 1565.83 \\
\hline Male & 0.86 & 0.79 & 1512.09 & 1522.66 & 0.85 & 0.77 & 1531.95 & $|542.5|$ \\
\hline \multicolumn{9}{|l|}{$\mathrm{C}+\mathrm{W}$} \\
\hline Female & 0.89 & 0.83 & 1434.26 & | 444.83 & 0.79 & 0.72 & I554.79 & I565.35 \\
\hline Male & 0.86 & 0.79 & $|5||.3|$ & $|52| .88$ & 0.85 & 0.78 & 1522.96 & I533.53 \\
\hline
\end{tabular}

Abbreviations: $\mathrm{C}+\mathrm{Hp}$, chest + hip circumference; $\mathrm{C}+\mathrm{U}$, chest + umbilical level circumference; $\mathrm{C}+\mathrm{W}$, chest + waist circumference. 
Table 4 Sex- and age group-specific original regression and modified simple formula derived from modeling formulation group

\begin{tabular}{|c|c|c|c|c|}
\hline \multirow{2}{*}{$\frac{\text { Age }}{\text { Type }}$} & \multicolumn{2}{|l|}{$<60$ years } & \multicolumn{2}{|l|}{$\geq 60$ years } \\
\hline & Original equation & Simple equation & Original equation & Simple equation \\
\hline \multicolumn{5}{|l|}{ Chest } \\
\hline Female & $1.01(\mathrm{C})+0.39(\mathrm{H})-90.33$ & $\mathrm{I}(\mathrm{C})+(\mathrm{H} / 3)-80$ & $0.90(\mathrm{C})+0.43(\mathrm{H})-90.72$ & I (C) $+(\mathrm{H} / 3)-85$ \\
\hline Male & $1.12(\mathrm{C})+0.39(\mathrm{H})-100.4$ & $(\mathrm{C})+(\mathrm{H} / 3)-80$ & $1.05(\mathrm{C})+0.35(\mathrm{H})-91.95$ & I (C) $+(H / 3)-85$ \\
\hline \multicolumn{5}{|c|}{ 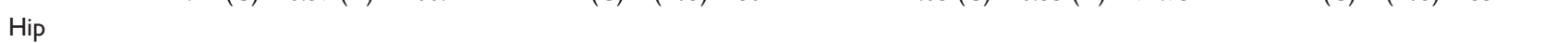 } \\
\hline Female & $\mathrm{I} .00(\mathrm{Hp})+0.32(\mathrm{H})-87.37$ & I $(H p)+(H / 3)-90$ & $0.76(\mathrm{Hp})+0.50(\mathrm{H})-93.08$ & $0.8(\mathrm{Hp})+(\mathrm{H} / 2)-95$ \\
\hline Male & $1.10(\mathrm{Hp})+0.36(\mathrm{H})-97.38$ & I $(H p)+(H / 3)-85$ & $0.81(H p)+0.49(H)-94.72$ & $0.8(\mathrm{Hp})+(\mathrm{H} / 2)-95$ \\
\hline \multicolumn{5}{|l|}{ Umbilical } \\
\hline Female & $0.83(\mathrm{U})+0.49(\mathrm{H})-86.46$ & $0.8(U)+(H / 2)-85$ & $0.58(\mathrm{U})+0.67(\mathrm{H})-97.00$ & $0.8(U)+(H / 2)-90$ \\
\hline Male & $0.85(\mathrm{U})+0.49(\mathrm{H})-85.42$ & $0.8(U)+(H / 2)-80$ & $0.77(\mathrm{U})+0.55(\mathrm{H})-96.20$ & $0.8(U)+(H / 2)-90$ \\
\hline \multicolumn{5}{|l|}{ Waist } \\
\hline Female & $0.90(\mathrm{~W})+0.48(\mathrm{H})-86.44$ & $I(W)+(H / 2)-95$ & $0.65(\mathrm{~W})+0.62(\mathrm{H})-92.69$ & $\mathrm{I}(\mathrm{W})+(\mathrm{H} / 2)-100$ \\
\hline Male & $0.89(\mathrm{~W})+0.50(\mathrm{H})-89.08$ & $I(W)+(H / 2)-100$ & $0.83(\mathrm{~W})+0.55(\mathrm{H})-98.93$ & $I(W)+(H / 2)-105$ \\
\hline \multicolumn{5}{|l|}{$\mathrm{C}+\mathrm{Hp}$} \\
\hline Female & $0.58(\mathrm{C}+\mathrm{Hp})+0.3 \mathrm{I}(\mathrm{H})-94.82$ & $0.6(\mathrm{C}+\mathrm{H} \mathrm{p})+(\mathrm{H} / 3)-100$ & $0.47(\mathrm{C}+\mathrm{Hp})+0.43(\mathrm{H})-96.47$ & $0.6(\mathrm{C}+\mathrm{Hp})+(\mathrm{H} / 3)-105$ \\
\hline Male & $0.65(\mathrm{C}+\mathrm{Hp})+0.3 \mathrm{I}(\mathrm{H})-107.05$ & $0.6(\mathrm{C}+\mathrm{Hp})+(\mathrm{H} / 3)-100$ & $0.57(\mathrm{C}+\mathrm{Hp})+0.3 \mathrm{I}(\mathrm{H})-96.34$ & $0.6(\mathrm{C}+\mathrm{Hp})+(\mathrm{H} / 3)-105$ \\
\hline \multicolumn{5}{|l|}{$\mathrm{C}+\mathrm{U}$} \\
\hline Female & $0.50(\mathrm{C}+\mathrm{U})+0.42(\mathrm{H})-91.70$ & $0.5(\mathrm{C}+\mathrm{U})+(\mathrm{H} / 2)-105$ & $0.41(\mathrm{C}+\mathrm{U})+0.55(\mathrm{H})-99.59$ & $0.5(\mathrm{C}+\mathrm{U})+(\mathrm{H} / 2)-110$ \\
\hline Male & $0.56(\mathrm{C}+\mathrm{U})+0.40(\mathrm{H})-97.75$ & $0.5(\mathrm{C}+\mathrm{U})+(\mathrm{H} / 2)-105$ & $0.52(\mathrm{C}+\mathrm{U})+0.40(\mathrm{H})-96.42$ & $0.5(\mathrm{C}+\mathrm{U})+(\mathrm{H} / 2)-110$ \\
\hline \multicolumn{5}{|l|}{$\mathrm{C}+\mathrm{W}$} \\
\hline Female & $0.5 \mathrm{I}(\mathrm{C}+\mathrm{W})+0.42(\mathrm{H})-91.06$ & $0.6(C+W)+(H / 3)-90$ & $0.43(\mathrm{C}+\mathrm{W})+0.5 \mathrm{I}(\mathrm{H})-96.24$ & $0.5(C+W)+(H / 2)-105$ \\
\hline Male & $0.56(\mathrm{C}+\mathrm{W})+0.4 \mathrm{I}(\mathrm{H})-99.5 \mathrm{I}$ & $0.6(C+W)+(H / 3)-95$ & $0.54(\mathrm{C}+\mathrm{W})+0.40(\mathrm{H})-98.07$ & $0.5(C+W)+(H / 2)-110$ \\
\hline
\end{tabular}

Abbreviations: $\mathrm{C}+\mathrm{Hp}$, chest + hip circumference; $\mathrm{C}+\mathrm{U}$, chest + umbilical level circumference; $\mathrm{C}+\mathrm{W}$, chest + waist circumference.

three formulas using chest, $\mathrm{C}+\mathrm{Hp}$, and $\mathrm{C}+\mathrm{U}$ had these properties (Table 4).

Model validity was tested in three aspect questions. First, which models between Sco and Cco were appropriate equations in term of precision? Second, do simple formulas have the similar prediction value comparing with original regression model? Third, which covariate equation should be recommended in Sco and Cco? For the first question, in addition to each formula, fittings were compared and verified using correlation coefficient (r), adjusted R-square, AIC, and BIC which were demonstrated in Table 3. They also were tested by absolute error difference (Table 5 and Figure 1). We observed that the Cco equations had more correlation coefficient and adjusted R-square as well as less AIC and BIC than the Sco equations which could be interpreted that Cco have had better model fitting than the Sco. In addition, performance of equations with each covariate prediction was tested. Absolute errors were compared and demonstrated as the differences of them within formula types comparing between single versus single (SS), combination versus combination (CC), and combination versus single covariate (CS) formulas; these were demonstrated in Figure 1 as varying shade colors of green (SS), blue (CC), and red (CS) bars, respectively. In Figure 1, SS and CC had comparable total evidence of significant comparison pairs to total pairs
(SS versus CC: $33.33 \%$ [16/48] versus 37.5\% [9/24]; $P=0.73$ ). However, there was significantly higher evidence of distinctly CS pairs than non-CS (CC and SS) pairs (CS versus non-CS: $69.79 \%$ [67/96] versus $34.72 \%$ [25/72]; $P<0.001$, respectively). These could be interpreted that comparison within the same type of Sco or Cco equations were comparable, but comparison between the different types of equation had significant difference errors. In addition, at the error threshold at $10 \%$ and $20 \%$ (Table 7 and Figure 4), the tolerance threshold of error in Cco had more accuracy than Sco (mean percentage error tolerance of Cco versus Sco [95\% confidence interval $(95 \% \mathrm{CI}) ; P$ value]: $10 \% ; 76.9$ versus 70.3 [74.2-79.6 versus $68.4-72.3 ; P<0.01]$ and $20 \% ; 96.8$ versus 94.5 [95.7-97.7 versus 93.2-95.8; $P<0.01]$ ). The subgroup analyses on sex, age group, and type of equations (Figure 4) also had corresponding results. Therefore, the Cco equations had more precision and error tolerance than Sco equations.

The second question aimed to compare the performance of original and modified simple formulas. The authors demonstrated these performance errors in two aspects. First, using critical error levels, which were determined into two thresholds of error and error tolerance at $10 \%$ and $20 \%$ (Tables 6, 7, and Figure 4). Second, quantitative errors of equation were demonstrated by Bland-Altman plot, in which each error value was located on their actual body weight (Figures 2 and 3). By 
Table 5 Validation of original regression and modified simple formula from validation group classified by sex and age groups

\begin{tabular}{|c|c|c|c|c|c|c|}
\hline \multirow[t]{2}{*}{ Parameter } & \multicolumn{3}{|c|}{ Original formula } & \multicolumn{3}{|c|}{ Simple formula } \\
\hline & $r^{*}$ & Error $(\mathbf{k g})^{\dagger}$ & RE (\%) ${ }^{\dagger}$ & $r^{*}$ & Error $(\mathbf{k g})^{\dagger}$ & RE (\%) ${ }^{\dagger}$ \\
\hline \multicolumn{7}{|c|}{ Age $<60$ years } \\
\hline \multicolumn{7}{|c|}{ Chest } \\
\hline Female & 0.87 & $3.65 \pm 3.71$ & $6.49 \pm 6.58$ & 0.87 & $3.73 \pm 3.72$ & $6.71 \pm 6.87$ \\
\hline Male & 0.84 & $4.69 \pm 4.16$ & $7.12 \pm 6.54$ & 0.84 & $4.87 \pm 4.15$ & $7.38 \pm 6.51$ \\
\hline \multicolumn{7}{|l|}{ Hip } \\
\hline Female & 0.86 & $4.27 \pm 3.50$ & $7.45 \pm 5.74$ & 0.86 & $4.38 \pm 3.63$ & $7.58 \pm 5.85$ \\
\hline Male & 0.86 & $4.58 \pm 3.70$ & $6.75 \pm 5.22$ & 0.86 & $4.83 \pm 4.05$ & $6.95 \pm 5.18$ \\
\hline \multicolumn{7}{|l|}{ Umbilical } \\
\hline Female & 0.87 & $4.11 \pm 3.22$ & $7.32 \pm 5.82$ & 0.87 & $4.07 \pm 3.18$ & $7.30 \pm 5.87$ \\
\hline Male & 0.87 & $4.57 \pm 3.48$ & $6.90 \pm 5.36$ & 0.87 & $5.37 \pm 3.90$ & $8.46 \pm 6.90$ \\
\hline \multicolumn{7}{|l|}{ Waist } \\
\hline Female & 0.88 & $3.84 \pm 3.05$ & $6.87 \pm 5.65$ & 0.89 & $4.30 \pm 3.42$ & $7.83 \pm 6.67$ \\
\hline Male & 0.87 & $4.46 \pm 3.42$ & $6.67 \pm 5.09$ & 0.87 & $4.68 \pm 3.71$ & $6.88 \pm 5.23$ \\
\hline \multicolumn{7}{|l|}{$\mathrm{C}+\mathrm{Hp}$} \\
\hline Female & 0.92 & $3.13 \pm 2.76$ & $5.59 \pm 4.99$ & 0.92 & $3.72 \pm 3.10$ & $6.78 \pm 5.97$ \\
\hline Male & 0.90 & $3.91 \pm 3.18$ & $5.77 \pm 4.53$ & 0.90 & $3.91 \pm 3.18$ & $5.92 \pm 4.89$ \\
\hline \multicolumn{7}{|l|}{$\mathrm{C}+\mathrm{U}$} \\
\hline Female & 0.91 & $3.37 \pm 2.82$ & $6.00 \pm 4.98$ & 0.91 & $3.56 \pm 2.91$ & $6.27 \pm 4.89$ \\
\hline Male & 0.89 & $4.05 \pm 3.29$ & $6.10 \pm 5.16$ & 0.89 & $4.09 \pm 3.49$ & $6.05 \pm 5.09$ \\
\hline \multicolumn{7}{|l|}{$\mathrm{C}+\mathrm{W}$} \\
\hline Female & 0.91 & $3.34 \pm 2.80$ & $5.94 \pm 4.94$ & 0.91 & $4.01 \pm 3.16$ & $7.25 \pm 6.01$ \\
\hline Male & 0.89 & $4.07 \pm 3.39$ & $6.02 \pm 4.99$ & 0.89 & $4.44 \pm 3.56$ & $6.51 \pm 5.07$ \\
\hline \multicolumn{7}{|c|}{ Age $\geq 60$ yrs } \\
\hline \multicolumn{7}{|c|}{ Chest } \\
\hline Female & 0.84 & $4.40 \pm 3.96$ & $7.98 \pm 6.47$ & 0.83 & $4.53 \pm 4.01$ & $8.24 \pm 6.68$ \\
\hline Male & 0.86 & $4.46 \pm 4.01$ & $7.58 \pm 6.67$ & 0.86 & $4.52 \pm 4.07$ & $7.63 \pm 6.66$ \\
\hline \multicolumn{7}{|l|}{ Hip } \\
\hline Female & 0.84 & $4.55 \pm 3.74$ & $8.74 \pm 7.40$ & 0.84 & $4.92 \pm 4.03$ & $9.73 \pm 8.66$ \\
\hline Male & 0.81 & $5.32 \pm 4.56$ & $9.31 \pm 8.26$ & 0.81 & $5.38 \pm 4.57$ & $9.49 \pm 8.45$ \\
\hline \multicolumn{7}{|l|}{ Umbilical } \\
\hline Female & 0.80 & $4.97 \pm 4.29$ & $9.34 \pm 8.17$ & 0.78 & $5.36 \pm 4.37$ & $10.17 \pm 8.74$ \\
\hline Male & 0.88 & $4.47 \pm 3.61$ & $7.54 \pm 5.94$ & 0.88 & $4.40 \pm 3.49$ & $7.52 \pm 6.03$ \\
\hline \multicolumn{7}{|l|}{ Waist } \\
\hline Female & 0.78 & $4.99 \pm 4.53$ & $9.41 \pm 8.57$ & 0.76 & $5.90 \pm 5.39$ & $11.50 \pm 11.17$ \\
\hline Male & 0.88 & $4.52 \pm 3.55$ & $7.67 \pm 6.06$ & 0.88 & $4.7 I \pm 3.45$ & $8.10 \pm 6.16$ \\
\hline \multicolumn{7}{|l|}{$\mathrm{C}+\mathrm{Hp}$} \\
\hline Female & 0.88 & $3.91 \pm 3.23$ & $7.38 \pm 6.10$ & 0.88 & $4.19 \pm 3.50$ & $7.98 \pm 6.84$ \\
\hline Male & 0.88 & $4.28 \pm 3.79$ & $7.28 \pm 6.26$ & 0.88 & $4.17 \pm 3.74$ & $7.22 \pm 6.45$ \\
\hline \multicolumn{7}{|l|}{$\mathrm{C}+\mathrm{U}$} \\
\hline Female & 0.85 & $4.34 \pm 3.58$ & $8.21 \pm 7.06$ & 0.85 & $5.01 \pm 3.83$ & $9.30 \pm 7.06$ \\
\hline Male & 0.91 & $3.75 \pm 3.13$ & $6.41 \pm 5.47$ & 0.91 & $3.87 \pm 3.31$ & $6.51 \pm 5.52$ \\
\hline \multicolumn{7}{|l|}{$\mathrm{C}+\mathrm{W}$} \\
\hline Female & 0.84 & $4.44 \pm 3.85$ & $8.25 \pm 6.94$ & 0.84 & $4.62 \pm 3.85$ & $8.76 \pm 7.59$ \\
\hline Male & 0.91 & $3.75 \pm 3.16$ & $6.49 \pm 5.73$ & 0.91 & $4.4 I \pm 3.54$ & $7.29 \pm 5.54$ \\
\hline
\end{tabular}

Notes: $* P<0.01$ all; ${ }^{\dagger}$ mean \pm SD.

Abbreviations: $r$, correlation coefficient; $\mathrm{RE}$, relative error; $\mathrm{C}+\mathrm{Hp}$, chest + hip circumference; $\mathrm{C}+\mathrm{U}$, chest + umbilical level circumference; $\mathrm{C}+\mathrm{W}$, chest + waist circumference; $\mathrm{SD}$, standard deviation.

critical error threshold of $10 \%$ and $20 \%$, almost all simple equations had a higher error than the original formula (Table 6). However, there were no differences between the types of equations in term of error tolerance in both critical levels (Table 7 and Figure 4C). All kappa agreement (Table 6) correlations of error occurrence between the original and simple formulas had higher than $50 \%$ in all paired formulas except the $\mathrm{C}+\mathrm{U}$ older female (0.43), C+W older male (0.47) in 10\% threshold, and waist of the younger male (0.33) in the $20 \%$ threshold. However, these pair error occurrences between original and 

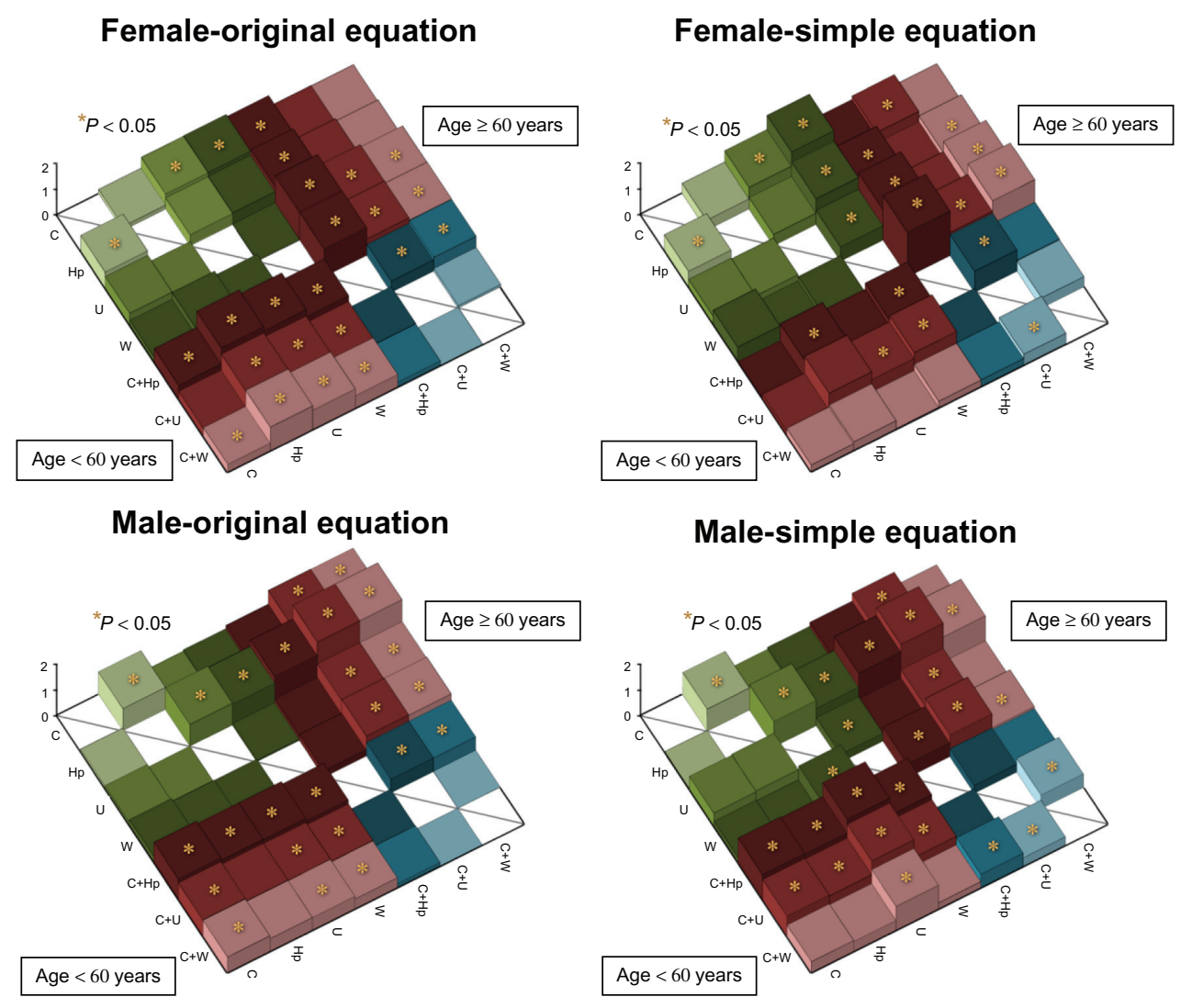

Single vs single covariate equation comparison pairs (SS)
Combination vs single covariate equation comparison pairs (CS)
Combination vs combination covariate equation comparison pairs (CC)

Figure I Absolute error difference between each covariate equation classified by type of formula, sex, and age groups.

Notes: Three-dimensional graphs show the comparison pattern of mean error difference between difference equation classified by equation type and sex. The diagonal line in each three-dimensional graph separates comparison performance within age group (above, $\geq 60$ years; below, $<60$ years). Green, red, and blue colors are the comparisons between single versus single (SS), combination versus single (CS), and combination versus combination covariate equation, respectively (CC). *Significant difference between models, $P<0.05$.

simple formulas had significant agreement with a $P$ value of less than 0.01 (Table 6). Of these agreements between original and simple equations, median agreement in the Sco equations was slightly higher than the Cco equations but there were no statistical differences (Median [interquartile range; IQR] Sco versus Cco: $10 \% ; 0.79$ [0.24] versus $0.66[0.20] ; P=0.14$ and $20 \% ; 0.74[0.13]$ versus $0.66[0.25] ; P=0.13)$. Subgroup analyses on sex and age group also had accorded results (female versus male: $10 \%$; 0.66 [0.19] versus 0.79 [0.24]; $P=0.24$ and $20 \% ; 0.72[0.14]$ versus $0.77[0.30] ; P=0.34$. Younger versus older: $10 \% ; 0.72$ [0.21] versus 0.74 [0.24]; $P=0.82$ and $20 \% ; 0.74$ [0.24] versus 0.74 [0.18]; $P=0.57$ ). Quantitative error over actual body weight using BlandAltman plots was demonstrated in Figure 2 (Sco equations) and Figure 3 (Cco equations). Of these figures, although most of prediction error was contained in two standard deviations, a negative correlation of error over actual body weight could be observed especially in the Sco equations and these correlations had more conversions to the baseline in Cco equations. However, in Figures 2 and 3, we could observe that both prediction formulas had the tendency to overestimation in lower body weights (less than $40 \mathrm{~kg}$ ) and underestimation in higher body weights (more than $90 \mathrm{~kg}$ ).

The third question was to select the appropriate equation by the anthropometric validation result criteria in a previous study which had around one-third occurrence on the total population of anthropometric body weight predicted formula at the $10 \%$ error threshold. ${ }^{10}$ With this criterion, acceptable performance equations were observed and selected depending on age group and sex as follows: first, in males, all Sco in both age groups could be included with this criterion, second, in females, selected equations were dependent on age groups. While all Sco could be selected in the younger female, only both chest Sco (original and simple; $31.2 \%$ and 
Table 6 Performance and error agreement between original regression and modified simple formula divided by sex and age group

\begin{tabular}{|c|c|c|c|c|c|c|}
\hline \multirow[t]{2}{*}{ Parameters } & \multicolumn{3}{|c|}{ Error $\geq 10 \%$} & \multicolumn{3}{|c|}{ Error $\geq 20 \%$} \\
\hline & Original $^{a}$ & Simple $^{a}$ & Kарра* & Original $^{\mathrm{a}}$ & Simple ${ }^{a}$ & Kappa* \\
\hline \multicolumn{7}{|c|}{ Age $<60$ years } \\
\hline \multicolumn{7}{|c|}{ Chest } \\
\hline Female & $51(20.4)$ & $54(21.6)$ & 0.89 & $7(2.8)$ & $12(4.8)$ & 0.73 \\
\hline Male & $62(24.8)$ & $66(26.4)$ & 0.85 & $10(4.0)$ & $12(4.8)$ & 0.81 \\
\hline \multicolumn{7}{|l|}{ Hip } \\
\hline Female & 77 (30.8) & 77 (30.8) & 0.85 & $5(2.0)$ & $8(3.2)$ & 0.61 \\
\hline Male & $63(25.2)$ & $66(26.4)$ & 0.78 & $7(2.8)$ & $4(1.6)$ & 0.72 \\
\hline \multicolumn{7}{|l|}{ Umbilical } \\
\hline Female & $72(28.8)$ & $68(27.2)$ & 0.80 & $6(2.4)$ & $8(3.2)$ & 0.85 \\
\hline Male & $60(24.0)$ & $83(33.2)$ & 0.53 & $8(3.2)$ & $14(5.6)$ & 0.72 \\
\hline \multicolumn{7}{|l|}{ Waist } \\
\hline Female & $52(20.8)$ & $65(26.0)$ & 0.61 & $9(3.6)$ & $12(4.8)$ & 0.85 \\
\hline Male & $60(24.0)$ & $69(27.6)$ & 0.53 & $4(1.6)$ & $2(0.8)$ & 0.33 \\
\hline \multicolumn{7}{|l|}{$\mathrm{C}+\mathrm{Hp}$} \\
\hline Female & $39(15.6)$ & $56(22.4)$ & 0.63 & $4(1.6)$ & $8(3.2)$ & 0.66 \\
\hline Male & $38(15.2)$ & $45(18.0)$ & 0.61 & $3(1.2)$ & $4(1.6)$ & 0.57 \\
\hline \multicolumn{7}{|l|}{$\mathrm{C}+\mathrm{U}$} \\
\hline Female & $42(16.8)$ & $44(17.6)$ & 0.72 & $5(2.0)$ & $5(2.0)$ & 0.59 \\
\hline Male & $47(18.8)$ & $47(18.8)$ & 0.82 & $3(1.2)$ & $3(1.2)$ & 1.00 \\
\hline \multicolumn{7}{|l|}{$\mathrm{C}+\mathrm{W}$} \\
\hline Female & $43(17.2)$ & $65(26.0)$ & 0.53 & $4(1.6)$ & $10(4.0)$ & 0.42 \\
\hline Male & $46(18.4)$ & $52(20.8)$ & 0.72 & $2(0.8)$ & $2(0.8)$ & 1.00 \\
\hline \multicolumn{7}{|c|}{ Age $\geq 60$ years } \\
\hline \multicolumn{7}{|c|}{ Chest } \\
\hline Female & $78(3 \mid .2)$ & $83(33.2)$ & 0.84 & $12(4.8)$ & $12(4.8)$ & 0.91 \\
\hline Male & $69(27.6)$ & $69(27.6)$ & 0.88 & $15(6.0)$ & $15(6.0)$ & 1.00 \\
\hline \multicolumn{7}{|l|}{ Hip } \\
\hline Female & $82(32.8)$ & 98 (39.2) & 0.76 & $21(8.4)$ & 32 (12.8) & 0.73 \\
\hline Male & $84(33.6)$ & $82(32.8)$ & 0.95 & $27(10.8)$ & $27(10.8)$ & 0.96 \\
\hline \multicolumn{7}{|l|}{ Umbilical } \\
\hline Female & $96(38.4)$ & 98 (39.2) & 0.61 & $24(9.6)$ & $25(10.0)$ & 0.75 \\
\hline Male & $75(30.0)$ & $70(28.0)$ & 0.85 & $3(3.6)$ & $10(4.0)$ & 0.84 \\
\hline \multicolumn{7}{|l|}{ Waist } \\
\hline Female & 91 (36.4) & $103(4 \mid .2)$ & 0.50 & $22(8.8)$ & 38 (I5.2) & 0.66 \\
\hline Male & $74(29.6)$ & 77 (30.8) & 0.71 & $10(4.0)$ & $12(4.8)$ & $0.7 \mathrm{I}$ \\
\hline \multicolumn{7}{|l|}{$\mathrm{C}+\mathrm{Hp}$} \\
\hline Female & $64(25.6)$ & $76(30.4)$ & 0.64 & $10(4.0)$ & $15(6.0)$ & $0.7 \mathrm{I}$ \\
\hline Male & $62(24.8)$ & $65(26.0)$ & 0.88 & $9(3.6)$ & $10(4.0)$ & 0.84 \\
\hline \multicolumn{7}{|l|}{$\mathrm{C}+\mathrm{U}$} \\
\hline Female & 78 (3।.2) & $97(38.8)$ & 0.43 & $15(6.0)$ & I8 (7.2) & 0.51 \\
\hline Male & $48(19.2)$ & $56(22.4)$ & 0.81 & $7(2.8)$ & $5(2.0)$ & 0.66 \\
\hline \multicolumn{7}{|l|}{$\mathrm{C}+\mathrm{W}$} \\
\hline Female & $83(33.2)$ & $82(32.8)$ & 0.67 & I4 (5.6) & $20(8.0)$ & 0.75 \\
\hline Male & 47 (18.8) & $65(26.0)$ & 0.47 & $9(3.6)$ & $6(2.4)$ & 0.52 \\
\hline
\end{tabular}

Notes: aNumber of error (\%), *kappa agreement $P$ value $<0.00 \mathrm{I}$ all of parameters.

Abbreviations: Kappa, kappa agreement probability; $\mathrm{C}+\mathrm{Hp}$, chest + hip circumference; $\mathrm{C}+\mathrm{U}$, chest + umbilical level circumference; $\mathrm{C}+\mathrm{W}$, chest + waist circumference.

$33.2 \%)$ and original hip Sco (32.8\%) in the older female could be included. Third, for Cco, all predicted formulas had this acceptable performance except in the $\mathrm{C}+\mathrm{U}$ simple equation in the elderly female (38.8\%). Of these results and quantitative error to actual body weight in Table 6 as well as error tolerance in Table 7, at the overall aspect, the appropriate chest containing equations of Sco in both sex and age groups had higher accuracy than other Sco in terms of error tolerance. (Chest versus non-Chest [95\% CI] 10\%: 73.4 [69.7-77.1] versus 69.3 [67.0-71.6]; $P=0.03 .20 \%: 95.3$ [93.2-95.8] versus 94.3 [92.6-96.0]; $P=0.25)$. In addition, the Sco using chest covariate equations had the highest kappa agreement between the original and simple formula. For the Cco equation, error and error tolerance were comparable (Tables 6, 7, and 
Table 7 Mean error tolerance threshold with 95\% confidence interval classified by sex, age groups, and types of equations

\begin{tabular}{|c|c|c|c|c|c|c|c|c|c|c|}
\hline \multirow{2}{*}{$\begin{array}{l}\text { Parameters } \\
\text { Mean }(95 \% \mathrm{Cl})\end{array}$} & \multicolumn{3}{|l|}{ Sex } & \multicolumn{3}{|l|}{ Age group } & \multicolumn{3}{|l|}{ Type } & \multirow[t]{2}{*}{ All } \\
\hline & Female & Male & $P$ & Younger & Older & $P$ & Original & Simple & & \\
\hline \multicolumn{11}{|l|}{$10 \%$ threshold } \\
\hline Chest & $\begin{array}{l}73.4 \\
(63.0-83.8)\end{array}$ & $\begin{array}{l}73.4 \\
(71.3-75.5)\end{array}$ & 1.00 & $\begin{array}{l}76.7 \\
(72.3-81.1)\end{array}$ & $\begin{array}{l}70.1 \\
(65.7-74.5)\end{array}$ & 0.02 & $\begin{array}{l}74 \\
(66.7-81.3)\end{array}$ & $\begin{array}{l}72.8 \\
(65.2-80.4)\end{array}$ & 0.73 & $\begin{array}{l}73.4 \\
(69.7-77.1)\end{array}$ \\
\hline Hip & $\begin{array}{l}66.6 \\
(60.3-72.9)\end{array}$ & $\begin{array}{l}70.5 \\
(63.6-77.4)\end{array}$ & 0.23 & $\begin{array}{l}71.7 \\
(67.0-76.4)\end{array}$ & $\begin{array}{l}65.4 \\
(60.5-70.3)\end{array}$ & 0.03 & $\begin{array}{l}69.4 \\
(63.4-75.4)\end{array}$ & $\begin{array}{l}67.7 \\
(59.2-76.2)\end{array}$ & 0.62 & $\begin{array}{l}68.55 \\
(64.9-72.2)\end{array}$ \\
\hline Umbilical & $\begin{array}{l}66.6 \\
(56.6-76.6)\end{array}$ & $\begin{array}{l}71.2 \\
(65.1-77.3)\end{array}$ & 0.26 & $\begin{array}{l}71.7 \\
(65.6-77.8)\end{array}$ & $\begin{array}{l}66.1 \\
(57.0-75.2)\end{array}$ & 0.16 & $\begin{array}{l}69.7 \\
(60.2-79.2)\end{array}$ & $\begin{array}{l}68.1 \\
(59.3-76.9)\end{array}$ & 0.71 & $\begin{array}{l}68.9 \\
(64.4-73.4)\end{array}$ \\
\hline Waist & $\begin{array}{l}68.9 \\
(54.0-83.8)\end{array}$ & $\begin{array}{l}72.0 \\
(67.3-76.7)\end{array}$ & 0.55 & $\begin{array}{l}75.4 \\
(70.7-80.1)\end{array}$ & $\begin{array}{l}65.5 \\
(57.0-74.0)\end{array}$ & 0.02 & $\begin{array}{l}72.3 \\
(61.4-83.2)\end{array}$ & $\begin{array}{l}68.6 \\
(57.7-79.5)\end{array}$ & 0.47 & $\begin{array}{l}70.4 \\
(64.9-76.0)\end{array}$ \\
\hline $\mathrm{C}+\mathrm{Hp}$ & $\begin{array}{l}76.5 \\
(66.6-86.4)\end{array}$ & $\begin{array}{l}79.0 \\
(70.7-87.3)\end{array}$ & 0.56 & $\begin{array}{l}82.2 \\
(76.9-87.5)\end{array}$ & $\begin{array}{l}73.3 \\
(69.3-77.3)\end{array}$ & $<0.01$ & $\begin{array}{l}79.7 \\
(70.7-88.7)\end{array}$ & $\begin{array}{l}75.8 \\
(67.4-84.2)\end{array}$ & 0.35 & $\begin{array}{l}77.6 \\
(73.2-82.3)\end{array}$ \\
\hline $\mathrm{C}+\mathrm{U}$ & $\begin{array}{l}73.9 \\
(56.8-91.0)\end{array}$ & $\begin{array}{l}80.2 \\
(77.4-83.0)\end{array}$ & 0.29 & $\begin{array}{l}82.0 \\
(80.4-83.6)\end{array}$ & $\begin{array}{l}72.1 \\
(58.0-86.2)\end{array}$ & 0.07 & $\begin{array}{l}78.5 \\
(68.1-88.9)\end{array}$ & $\begin{array}{l}75.6 \\
(60.0-91.2)\end{array}$ & 0.64 & $\begin{array}{l}77.0 \\
(70.5-83.6)\end{array}$ \\
\hline $\mathrm{C}+\mathrm{W}$ & $\begin{array}{l}72.7 \\
(60.8-84.6)\end{array}$ & $\begin{array}{l}79.0 \\
(73.4-84.6)\end{array}$ & 0.18 & $\begin{array}{l}79.4 \\
(73.2-85.6)\end{array}$ & $\begin{array}{l}72.3 \\
(61.5-83.1)\end{array}$ & 0.12 & $\begin{array}{l}78.1 \\
(66.1-90.1)\end{array}$ & $\begin{array}{l}73.6 \\
(65.8-81.4)\end{array}$ & 0.36 & $\begin{array}{l}75.9 \\
(70.5-81.2)\end{array}$ \\
\hline All & $\begin{array}{l}71.2 \\
(68.3-74.2)\end{array}$ & $\begin{array}{l}75.0 \\
(73.1-77.0)\end{array}$ & 0.03 & $\begin{array}{l}77.0 \\
(75.1-78.9)\end{array}$ & $\begin{array}{l}69.3 \\
(67.0-71.5)\end{array}$ & $<0.01$ & $\begin{array}{l}74.5 \\
(71.9-77.1)\end{array}$ & $\begin{array}{l}71.7 \\
(69.2-74.3)\end{array}$ & 0.12 & $\begin{array}{l}73.1 \\
(71.4-74.9)\end{array}$ \\
\hline \multicolumn{11}{|l|}{$20 \%$ threshold } \\
\hline Chest & $\begin{array}{l}95.7 \\
(94.1-97.3)\end{array}$ & $\begin{array}{l}94.8 \\
(93.2-96.5)\end{array}$ & 0.25 & $\begin{array}{l}95.9 \\
(94.4-97.4)\end{array}$ & $\begin{array}{l}94.6 \\
(93.5-95.7)\end{array}$ & 0.07 & $\begin{array}{l}95.6 \\
(93.5-97.7)\end{array}$ & $\begin{array}{l}94.9 \\
(93.9-95.8)\end{array}$ & 0.38 & $\begin{array}{l}95.3 \\
(94.4-96.1)\end{array}$ \\
\hline Hip & $\begin{array}{l}93.4 \\
(85.5-100)\end{array}$ & $\begin{array}{l}93.5 \\
(85.6-100)\end{array}$ & 0.98 & $\begin{array}{l}97.6 \\
(96.4-98.8)\end{array}$ & $\begin{array}{l}89.3 \\
(86.4-92.2)\end{array}$ & $<0.01$ & $\begin{array}{l}94.0 \\
(87.2-100)\end{array}$ & $\begin{array}{l}92.9 \\
(84.1-100)\end{array}$ & 0.76 & $\begin{array}{l}93.45 \\
(89.6-97.3)\end{array}$ \\
\hline Umbilical & $\begin{array}{l}93.7 \\
(87.2-100)\end{array}$ & $\begin{array}{l}95.9 \\
(94.2-97.6)\end{array}$ & 0.33 & $\begin{array}{l}96.4 \\
(94.2-98.6)\end{array}$ & $\begin{array}{l}93.2 \\
(87.7-98.7)\end{array}$ & 0.14 & $\begin{array}{l}95.3 \\
(90.0-100)\end{array}$ & $\begin{array}{l}94.3 \\
(89.5-99.1)\end{array}$ & 0.67 & $\begin{array}{l}94.8 \\
(92.3-97.3)\end{array}$ \\
\hline Waist & $\begin{array}{l}91.9 \\
(83.6-100)\end{array}$ & $\begin{array}{l}97.2 \\
(94.2-100)\end{array}$ & 0.11 & $\begin{array}{l}97.3 \\
(94.4-100)\end{array}$ & $\begin{array}{l}91.8 \\
(83.7-99.9)\end{array}$ & 0.09 & $\begin{array}{l}95.5 \\
(90.6-100)\end{array}$ & $\begin{array}{l}93.6 \\
(83.8-100)\end{array}$ & 0.60 & $\begin{array}{l}94.6 \\
(90.7-98.4)\end{array}$ \\
\hline $\mathrm{C}+\mathrm{Hp}$ & $\begin{array}{l}96.3 \\
(93.4-99.2)\end{array}$ & $\begin{array}{l}97.4 \\
(95.2-99.6)\end{array}$ & 0.38 & $\begin{array}{l}98.1 \\
(96.7-99.5)\end{array}$ & $\begin{array}{l}95.6 \\
(93.9-97.3)\end{array}$ & 0.01 & $\begin{array}{l}97.4 \\
(95.2-99.6)\end{array}$ & $\begin{array}{l}96.3 \\
(93.4-99.2)\end{array}$ & 0.38 & $\begin{array}{l}96.8 \\
(95.5-98.2)\end{array}$ \\
\hline $\mathrm{C}+\mathrm{U}$ & $\begin{array}{l}95.7 \\
(91.4-100)\end{array}$ & $\begin{array}{l}98.2 \\
(97.0-99.4)\end{array}$ & 0.13 & $\begin{array}{l}98.4 \\
(97.7-99.1)\end{array}$ & $\begin{array}{l}95.5 \\
(91.5-99.5)\end{array}$ & 0.06 & $\begin{array}{l}97.0 \\
(93.7-100)\end{array}$ & $\begin{array}{l}96.9 \\
(92.5-100)\end{array}$ & 0.96 & $\begin{array}{l}96.9 \\
(95.0-98.8)\end{array}$ \\
\hline $\mathrm{C}+\mathrm{W}$ & $\begin{array}{l}95.2 \\
(90.9-99.5)\end{array}$ & $\begin{array}{l}98.1 \\
(95.9-100)\end{array}$ & 0.10 & $\begin{array}{l}98.2 \\
(95.8-100)\end{array}$ & $\begin{array}{l}95.1 \\
(91.2-99.0)\end{array}$ & 0.08 & $\begin{array}{l}97.1 \\
(93.7-100)\end{array}$ & $\begin{array}{l}96.2 \\
(91.3-100)\end{array}$ & 0.65 & $\begin{array}{l}96.6 \\
(94.5-98.7)\end{array}$ \\
\hline All & $\begin{array}{l}94.6 \\
(93.2-95.9)\end{array}$ & $\begin{array}{l}96.4 \\
(95.4-97.4)\end{array}$ & 0.02 & $\begin{array}{l}97.4 \\
(96.9-98.0)\end{array}$ & $\begin{array}{l}93.6 \\
(92.3-94.9)\end{array}$ & $<0.01$ & $\begin{array}{l}96.0 \\
(95.0-97.0)\end{array}$ & $\begin{array}{l}93.6 \\
(94.6-96.4)\end{array}$ & 0.26 & $\begin{array}{l}95.5 \\
(94.7-96.3)\end{array}$ \\
\hline
\end{tabular}

Note: Error tolerance (\%) $=100-$ error (\%).

Abbreviations: $\mathrm{C}+\mathrm{Hp}$, chest + hip; $\mathrm{C}+\mathrm{U}$, chest + umbilical; $\mathrm{C}+\mathrm{W}$, chest + waist circumference.

Figure 4). Although there were no differences of error tolerance between the $\mathrm{C}+\mathrm{Hp}$ and other $\mathrm{Cco}$ equations $(\mathrm{C}+\mathrm{Hp}$ versus Non-C+Hp [95\% CI] 10\%: 77.8 [73.2-82.3] versus 76.5 [72.7-80.2]; $P=0.65 .20 \%: 96.9$ [95.5-98.2] versus 96.8 [95.6-98.0]; $P=0.96$ ), but $\mathrm{C}+\mathrm{Hp}$ had more error tolerance. In addition, we observed that $\mathrm{C}+\mathrm{Hp}$ had more precision and slightly higher mean error tolerance compared with other Cco in all subgroups (Figure 4, Table 7).

\section{Discussion}

Although weight scales are highly available, there were some limitations in special groups of people and many previous studies have suggested equations using anthropometric measurement to predict these parameters (Table 7). ${ }^{10-12,14-16,18}$ However, all of the population studies were collected and generated formulas based on the Western population and there were no suggested equations in the Asian population. Therefore, the present study was a pioneering endeavor to develop equations to predict body weight by anthropometric circumferential measurements. The present study separated equations divided by age groups and sex due to previous reports of variations of body composition depending on the age spectrum and sex difference and possibly interference to equation validity. ${ }^{17}$ These were demonstrated by differences of coefficient and intercepts at the same covariate equations in different age spectrums in the present study (Table 4). No differences were found in all of the measuring parameters between the modeling and validation groups (Table 1). In the selection process, stepwise regression analysis revealed multicollinearity between hip, umbilical level, and waist 

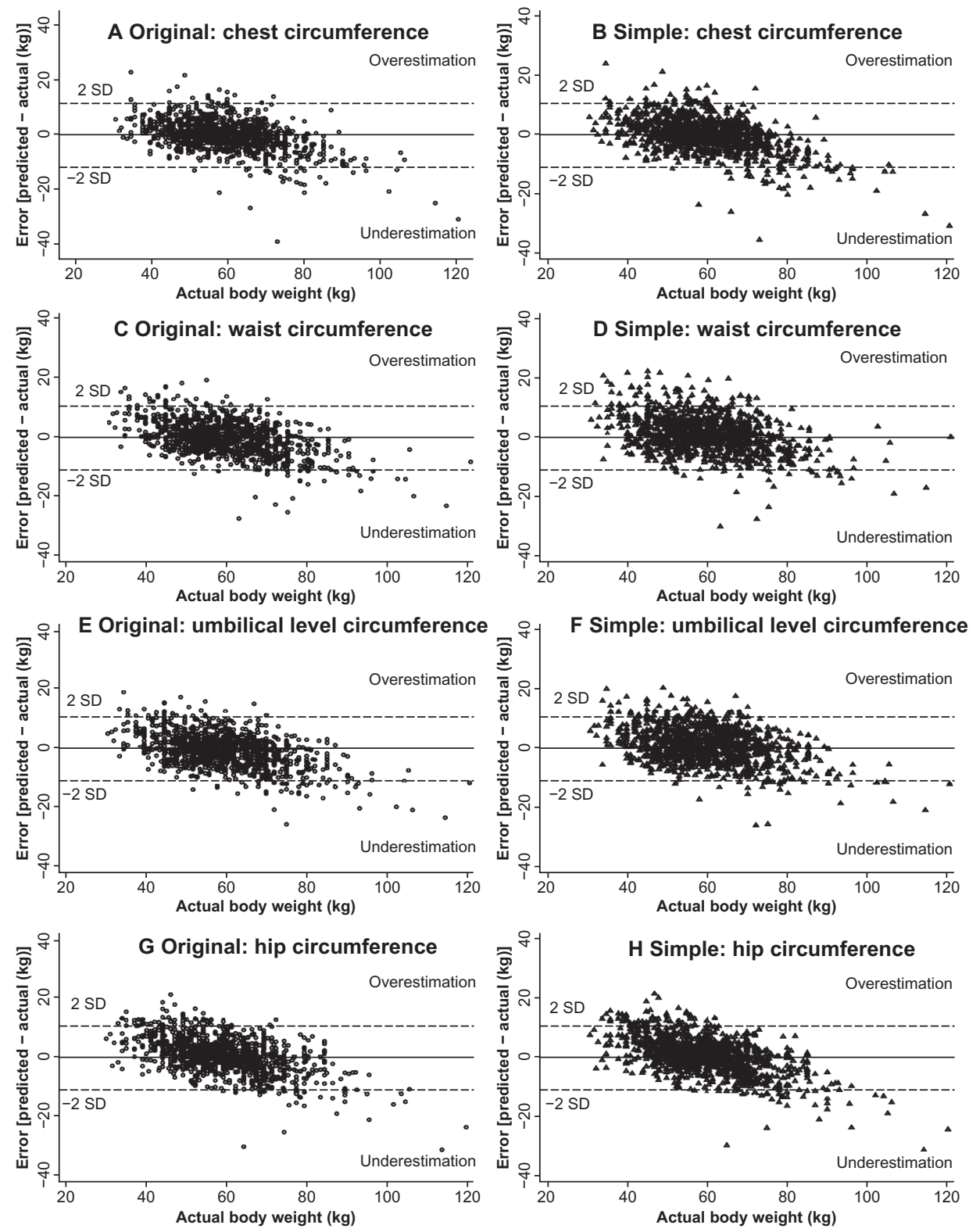

Figure 2 Bland-Altman plot between error of prediction and actual body weight in single covariate equations. Abbreviation: SD, standard deviation.

variables. Therefore, these covariates were separated into individual equations in the present study and the fixed covariate parameter of the individual equations was body height. Differences of body somatotype effect and body shape might affect the predictive validity. ${ }^{19-21}$ Although the authors did not detail body somatotype classifications due to the complexity of measurement, simple combinations between chest and one torso region were initiated by summation between the chest and abdominal region circumference (Cco) and these equations were tested for validation.

Previous studies using mid-arm and calf circumference together with skinfold thickness parameters in elderly people or using only arm circumference and height in obese people were proposed for body weight prediction. ${ }^{11,14-16,18}$ However, with our criteria for covariate selection, the authors found that mid-arm, mid-thigh, and mid-calf circumference had fewer fitting properties using correlation coefficient, adjusted R-square, AIC, and BIC than the other covariates (Table 2). Therefore, these variables were not selected for our model creation and validation processes. The probable reasons of these differences might be explained by different ethnic groups having different body composition as well as weight distribution. ${ }^{22}$ In addition, there were no comparisons between different circumferences of anthropometric measurements in previous studies. ${ }^{11,14-16,18}$ There were some concerns about the measurement difficulties of these torso 

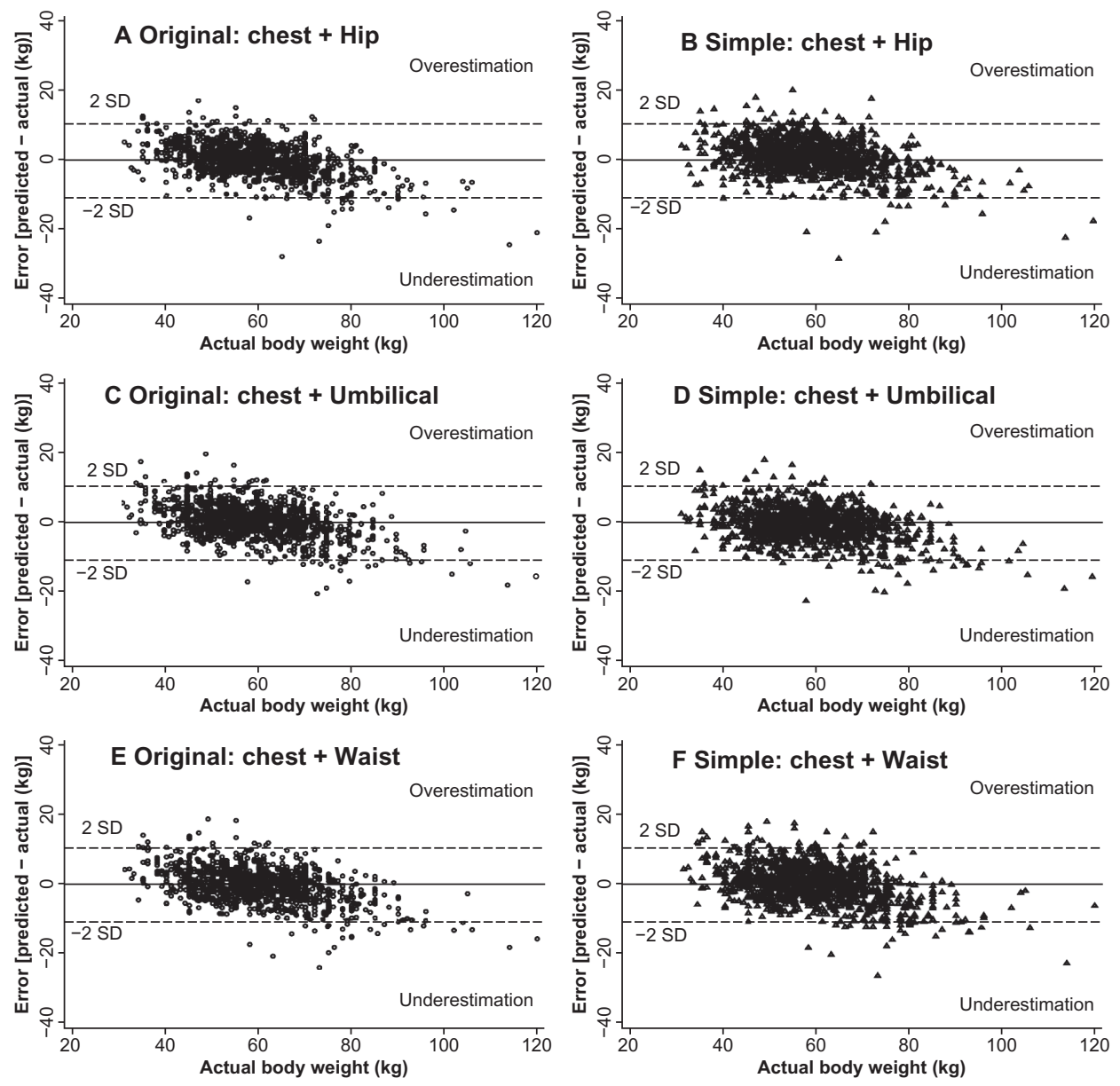

Figure 3 Bland-Altman plot between error and actual body weight in combination covariate equations.

Abbreviation: SD, standard deviation.

parameters, but these parameters might be collected and are feasible to perform during health care processes. The author divided the proposed models into two groups as mentioned previously: Sco equations and Cco equations. To simplify our equation for the purpose of bedside use, the covariate coefficients and constant intercepts were adjusted to simple numbers (Table 4). Both original and simple models were verified for validity to the other samples in the validation groups.

For the validation results in Table 5, both original and simple formulas had significant correlation coefficients of more than $0.75(P<0.01)$. Of these, the original and simple equations were comparable in correlation coefficients. Although there were comparable numbers of significant differences when comparing between CC and SS pairs, CS pairs had significant differences (Figure 1). When considered together with Tables 5 and 6 , these findings demonstrated that combination covariate equations had more precision than the single covariate equation. These might be explained by different somatotypes in volunteers. Somatotype patterns of endomorphic, mesomorphic, or ectomorphic body types are the important factor to determine different body circumference proportions and body stature. Different gender, age, and lifestyles lead to these somatotype distinctions. ${ }^{19,21}$ In the authors' opinion, summation of chest and one of torso circumference might simply be a method to decline the somatotype effect and these resulted in higher error tolerance in combination covariate equations. For the Sco equation (Tables 5, 6, and Figure 2) and Cco equation (Tables 5, 6, and Figure 3), even though chest-containing equations and the summation of chest and hip did not have the least predictive error in all age groups and sex, they had acceptable performance compared with all of the others. In addition, both equations had the better error tolerance when comparing the same type of equation in Figure 4. Therefore, the authors proposed these two equations to predict the actual body weight. The background reason to explain these findings was unknown, but the authors suspected that these variables had less variation and conformed alterations to body weight, body composition, and stature throughout life span. ${ }^{17,19}$ 


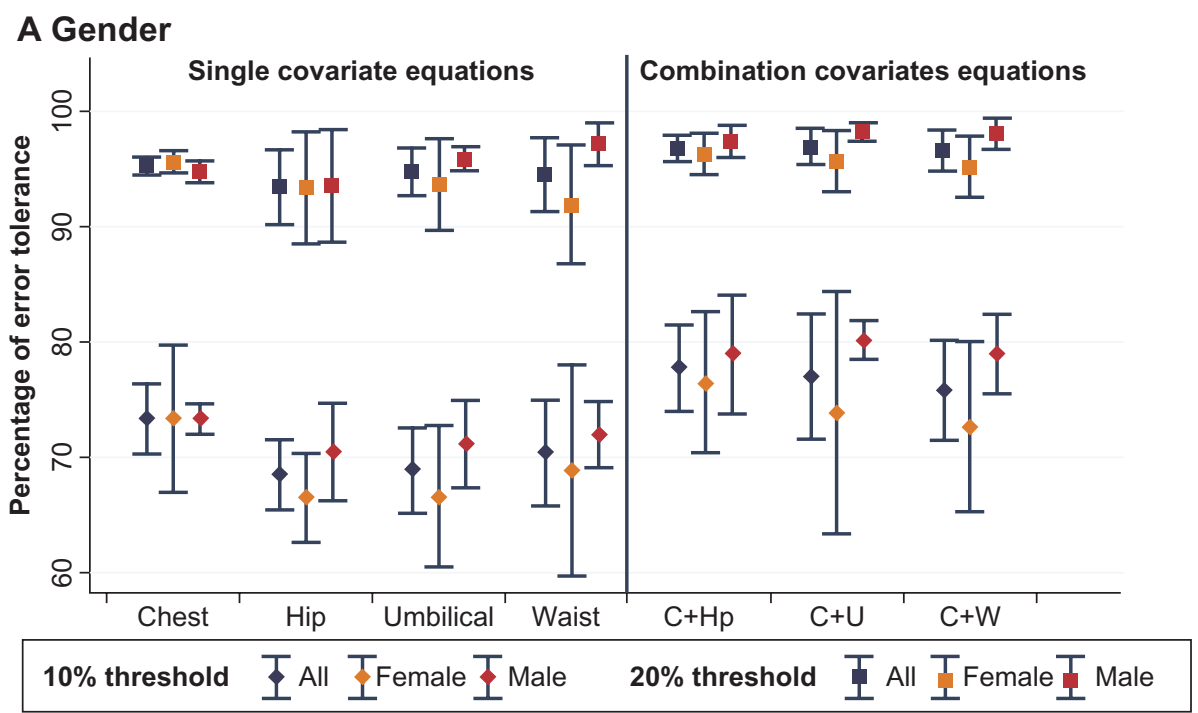

\section{B Age groups}

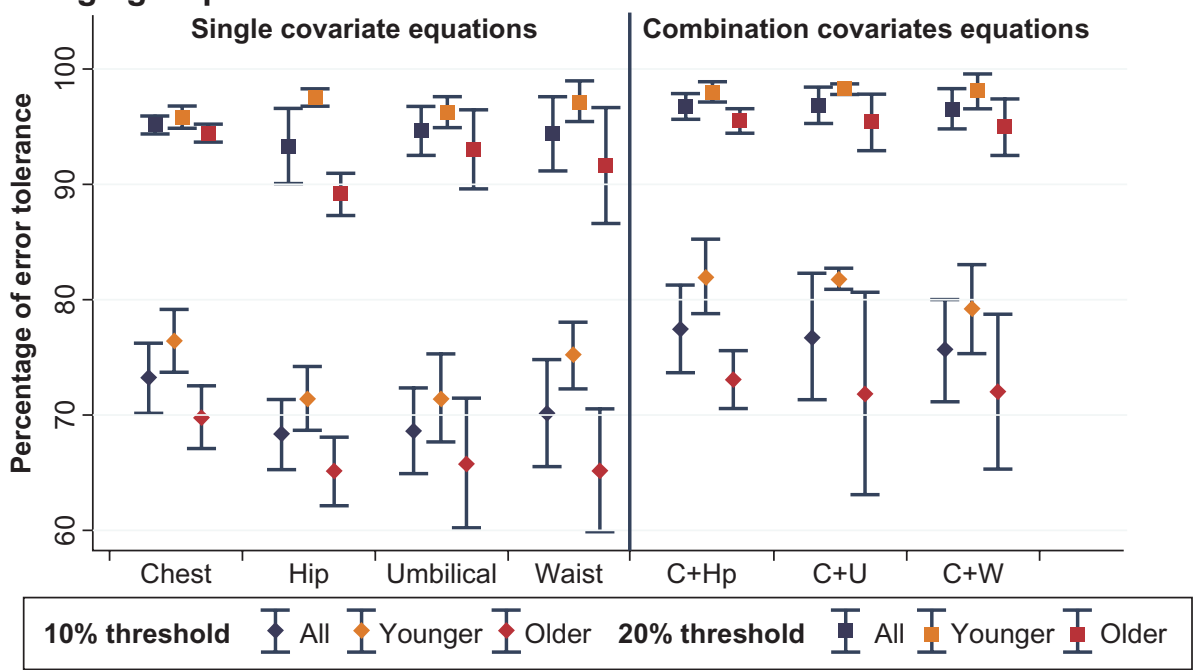

\section{Type of equations}

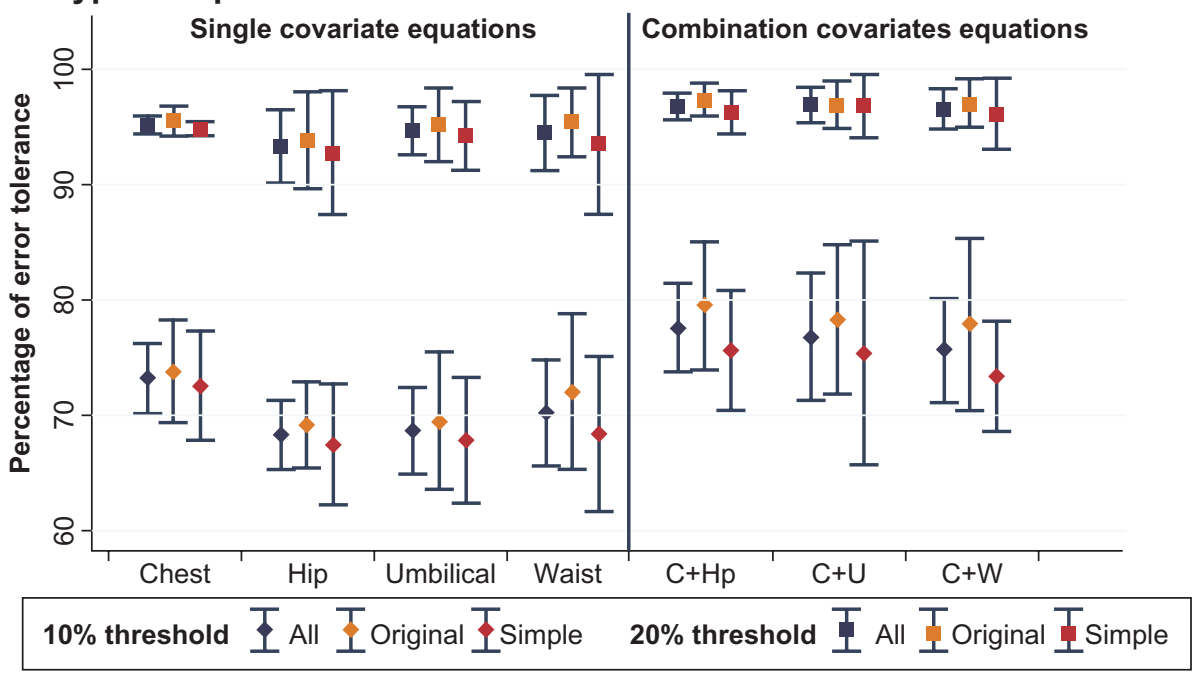

Figure 4 Percentage of error tolerance comparison in 10\% and $20 \%$ error threshold in each covariate equation classified by the subgroups (A) sex (B) age groups, and (C) type of equations.

Note: Error tolerance (\%) $=100-$ error (\%)

Abbreviations: $\mathrm{C}+\mathrm{Hp}$, chest + hip; $\mathrm{C}+\mathrm{U}$, chest + umbilical; $\mathrm{C}+\mathrm{W}$, chest + waist. 
Table 8 Summary of previous body weight predicted equation and validation

\begin{tabular}{|c|c|c|c|c|}
\hline Author & Population & Equation & Model & Validation \\
\hline Chumlea $^{16}$ & $\begin{array}{l}228 \text { elderly } \\
\text { (P, USA) }\end{array}$ & $\begin{array}{l}\text { Female: } \mathrm{WT}=0.98(\mathrm{MAC})+\mathrm{I} .27(\mathrm{CC})+0.40(\mathrm{SST})+ \\
0.87(\mathrm{KH})-62.35 \\
\text { Male: } \mathrm{WT}=1.73(\mathrm{MAC})+0.98(\mathrm{CC})+0.37(\mathrm{SST})+ \\
\mathrm{I} .16(\mathrm{KH})-81.69\end{array}$ & $\begin{array}{l}\text { Female } \\
R^{2}=0.85 \\
\text { Male } \\
R^{2}=0.90\end{array}$ & $\begin{array}{l}\text { Mean signed } \\
\text { differences } 0.1-1.8 \mathrm{~kg}\end{array}$ \\
\hline Donini $^{14}$ & $\begin{array}{l}285 \text { elderly } \\
(\mathrm{H}, \text { Italy) }\end{array}$ & $\begin{array}{l}\text { Female: } \mathrm{WT}=1.4 \mathrm{I}(\mathrm{MAC})+\mathrm{I} . \mathrm{I} \mathrm{I}(\mathrm{CC})+0.47(\mathrm{SST})+ \\
\text { I.0 }(\mathrm{KH})-67.37 \\
\text { Male: } \mathrm{WT}=36.2(\ln \mathrm{MAC})+42.47(\ln \mathrm{CC})+ \\
6.91(\ln \mathrm{SST})+0.8(\mathrm{KH})-253.7\end{array}$ & $\begin{array}{l}\text { Female } \\
R^{2}=0.83 \\
\text { Male } \\
R^{2}=0.89\end{array}$ & $\begin{array}{l}95 \% \text { error range } \\
\text { Woman: } \pm 6.1 \mathrm{~kg} \\
\text { Male: } \pm 4.9 \mathrm{~kg}\end{array}$ \\
\hline Jung ${ }^{18}$ & $\begin{array}{l}300 \text { elderly } \\
\text { (P+H, Hong Kong) }\end{array}$ & $\begin{array}{l}\text { Female: } \mathrm{WT}=1.0 \mathrm{I}(\mathrm{KH})+2.8 \mathrm{I}(\mathrm{MAC})-66.04 \\
\text { Male: } \mathrm{WT}=1.10(\mathrm{KH})+3.07(\mathrm{MAC})-75.8 \mathrm{I}\end{array}$ & See note ${ }^{a}$ & $\begin{array}{l}\text { Difference }(95 \% \mathrm{Cl}) \\
\text { Female: } 2.7(2.3 / 3.6) \\
\text { Male: } 0.4(-0.5 / \mathrm{I} .4)\end{array}$ \\
\hline Miyatake $^{13}$ & $\begin{array}{l}2635 \text { adults } \\
(\mathrm{H}, \text { Japan })\end{array}$ & $\begin{array}{l}\text { Female: } \downarrow 3 \mathrm{~kg} \approx \downarrow 2.85 \text { waist }(\mathrm{cm}) \\
\text { Male: } \downarrow 3 \mathrm{~kg} \approx \downarrow 3.45 \text { waist }(\mathrm{cm})\end{array}$ & NA & NA \\
\hline Crandall" & $\begin{array}{l}\text { I47| Obese } \\
(\mathrm{P}+\mathrm{H}, \text { USA })\end{array}$ & $\begin{array}{l}\text { Female: } \mathrm{WT}=2.15(\mathrm{MAC})+0.54(\mathrm{HT})-64.6 \\
\text { Male: } \mathrm{WT}=3.29(\mathrm{MAC})+0.43(\mathrm{HT})-93.2\end{array}$ & $\begin{array}{l}R^{2}=0.55 \\
R^{2}=0.59\end{array}$ & $\begin{array}{l}\text { Error } 10 \%: 30 \%-35 \% \\
\text { Error } 20 \%: 8 \%-10 \%\end{array}$ \\
\hline $\operatorname{Lin}^{10}$ & $\begin{array}{l}235 \text { adults } \\
(P, \text { USA })\end{array}$ & $\begin{array}{l}\text { Female: } \mathrm{WT}=1.0 \mathrm{I}(\mathrm{KH})+2.8 \mathrm{I}(\mathrm{MAC})-66.04 \\
\text { Male: } \mathrm{WT}=1.10(\mathrm{KH})+3.07(\mathrm{MAC})-75.8 \mathrm{I}\end{array}$ & See note ${ }^{a}$ & $\begin{array}{l}\text { Error } 10 \%: 31 \% \\
(95 \% \mathrm{Cl}: 25 \% / 37 \%)^{b}\end{array}$ \\
\hline Fawzy ${ }^{12}$ & $\begin{array}{l}50 \text { young male } \\
(\mathrm{H}, \text { Egypt })\end{array}$ & Male: $\mathrm{WT}=9.05(\mathrm{FBBL})+\mathrm{I} \mathrm{I} .53$ & $R^{2}=0.27$ & NA \\
\hline $\begin{array}{l}\text { Bernal- } \\
\text { Orozco }\end{array}$ & $\begin{array}{l}95 \text { elderly female } \\
\text { (P, Mexico) }\end{array}$ & $\begin{array}{l}\text { Female: } \mathrm{WT}=1.599(\mathrm{KH})+\mathrm{I} .135(\mathrm{MAC})+ \\
0.735(\mathrm{CC})+0.621(\mathrm{TSF})-83.123\end{array}$ & $R^{2}=0.90$ & $\begin{array}{l}\text { Difference error in } \\
\text { three samples: }-0.02 \pm 4.3 \\
-0.7 \pm 4.2 ; 1.9 \pm 3.2\end{array}$ \\
\hline
\end{tabular}

Notes: aStudy used Ross Laboratories equation (Columbus, $\mathrm{OH}$ ) for body weight prediction. These formulas were generated based on Caucasian population; ${ }^{\mathrm{b}}$ calculated from error tolerance.

Abbreviations: $\mathrm{H}$, healthy volunteers; $\mathrm{P}$, patients; WT, predicted body weight; $\mathrm{HT}$, height; $\mathrm{FBBL}$, left foot breadth at ball (measured by foot print method); $\mathrm{KH}$, knee height; MAC, mid arm circumference; CC, calf circumference; TSF, triceps skinfold thickness; SST, subscapular skinfold thickness; In, natural logarithm; NA, not available.

The most common body weight prediction method is visual estimation. However, many previous studies demonstrated that this was a poor estimation method and it was estimator-dependent. ${ }^{6,7,23}$ One prospective study in an intensive care unit demonstrated that body weight errors estimation of $\geq 10 \%$ and $\geq 20 \%$ of actual body weight were as much as $47 \%$ and $19 \%$, respectively. ${ }^{23}$ Although there were differences in the setting and population in the previous study, the single (Chest) and combination $(\mathrm{C}+\mathrm{Hp})$ proposed equations in this present study could decrease evidence errors predictions compared to a previous study ( $\geq 10 \%$ and $\geq 20 \%$ error [95\% CI]: Chest 26.6 [22.9-35.1] and 4.7 [3.9-5.6]. C+Hp 22.4 [17.7-29.5] and 3.2 [1.8-4.5], respectively; Table 6).

There were a number of potential strengths and weakness in the present study. The major strengths in the present study were a large sample size which was divided by gender and age groups. In addition, the modeling or development of the equation and validation groups were comparable in all basic demographics and measured data and different circumferences have been compared and demonstrate model fitting in the present study. However, there were a number of inevitable limitations for the study weakness. First, almost all of the participants in the present sample resided in the northern region of Thailand. Although all were the same ethnic background, the differences in lifestyle and living patterns between regions and Asian countries might affect the average body composition resulting in a validity distortion. However, the mixed ethnicity in the northern region of Thailand results from its geographic location between multiple nationalities. This might be the supportive factor to reduce ethnic differences when prediction results are extrapolated to other Asian countries. Second, nearly $80 \%$ of the volunteers had a body mass index of less than $25 \mathrm{~kg} / \mathrm{m}^{2}$, which is the criterion threshold of obesity diagnosis. Violations of linearity were performed before the model creation, but the authors found that underestimation might have occurred in volunteers with an actual body weight of more than $90 \mathrm{~kg}$ (Figures 2 and 3). Therefore, using the proposed prediction equation on this special population might include a caution for underestimating body weight. However, the present study demonstrated that using a combination covariate equation might alleviate these effects. The mathematic method to take the logarithm of covariate parameters before substitution of these values in the equations might diminish these effects and these methods have been proposed in a previous report (Table 7). ${ }^{14}$ 
However; simple formulation and general clinical bedside calculation were our primary aims. Therefore, logarithmbased models were not proposed in the present study. Third, although the authors attempted to decrease the somatotype effect by a simplifying method using the summation of chest and one torso circumference as one covariate in the equations, the actual somatotype-detailing anthropometric measurement data was not collected in the present study. Therefore, the correlation between these simplified methods and actual somatotype could not be demonstrated. However, the authors observed that these methods could reduce performance error when comparing individual covariatepredicted equations and further study might be performed to reveal the relationship between combination covariate and somatotypes. Fourth, because of internal validity concerns, the study population was collected only in healthy volunteers. Therefore, the equation results might be distorted when equations are extrapolated to diseased patients. However, there were inconsistent population recruitments in the previous studies of body weight prediction (Table 7). In addition, the actual body weight in diseased patients might deviate from functional body weight in healthy volunteers by body composition alternations. ${ }^{24,25}$ However, most phase I clinical trials were performed in healthy volunteers to determine the metabolic and pharmacological actions and the maximally tolerated dose. Of these backgrounds, in the authors' opinions, functional body weight from healthy volunteers might be applied to general clinical practice. Because of these limitations, further validation studies should be performed using these equations in the special clinical situations of the emergency department, intensive care units, or with immobilized patients. Finally, the authors proposed simple formulas which could be used in both sexes in the same age group. Although the authors endeavored to titrate the regression coefficient and intercept by substitution covariates with the mean value of the modeling sample as well as comparable correlation coefficients, the performance error of these equations was higher than the original ones. However, error tolerances of simple and original equations had comparable evidences of accuracy in the $10 \%$ error threshold range from one-fourth to one-third of the total sample. Therefore, these methods should be used only in situations in which a direct measurement is unavailable.

\section{Conclusion}

Body weight might be predicted by height and circumferential covariates equations. Cco had more error tolerance than
Sco. Original and simple equations had comparable validity. Chest- and $\mathrm{C}+\mathrm{Hp}$-containing covariate equations had more precision between Sco and Cco equations, respectively.

\section{Acknowledgments}

This study was supported by the Medical Association of Thailand (Prasert Prasarttong-Osoth) Research Fund. The authors gratefully thank the surgical outpatient department and hospital registration unit of the Maharaj Nakorn Chiang Mai Hospital, Faculty of Medicine, Chiang Mai University, Chiang Mai, Thailand for providing rooms and assistance.

\section{Disclosure}

The authors report no conflicts of interest in this work. This study was a part of thesis in the clinical epidemiology $\mathrm{PhD}$ project of Assistant Professor Kaweesak Chittawatanarat.

\section{References}

1. Sabol VK. Nutrition assessment of the critically ill adult. AACN Clin Issues. 2004;15(4):595-606.

2. Gehan EA, George SL. Estimation of human body surface area from height and weight. Cancer Chemother Rep. 1970;54(4):225-235.

3. Luscombe M, Owens B. Weight estimation in resuscitation: is the current formula still valid? Arch Dis Child. 2007;92(5):412-415.

4. Gunther A, Taut F. Tidal volume in mechanical ventilation: the importance of considering predicted body weight. Am J Respir Crit Care Med. 2008;178(3):315-316; author reply, 316.

5. Goutelle S, Bourguignon L, Bertrand-Passeron N, Jelliffe RW, Maire P. Visual estimation of patients' body weight in hospital: the more observers, the better? Pharm World Sci. 2009;31(4):422-425.

6. Anglemyer BL, Hernandez C, Brice JH, Zou B. The accuracy of visual estimation of body weight in the ED. Am J Emerg Med. 2004;22(7): 526-529.

7. Coe TR, Halkes M, Houghton K, Jefferson D. The accuracy of visual estimation of weight and height in pre-operative supine patients. Anaesthesia. 1999;54(6):582-586.

8. Leary TS, Milner QJ, Niblett DJ. The accuracy of the estimation of body weight and height in the intensive care unit. Eur J Anaesthesiol. 2000;17(11):698-703.

9. dos Reis Macedo LG, de Oliveira L, Pintao MC, Garcia AA, Pazin-Filho A. Error in body weight estimation leads to inadequate parenteral anticoagulation. Am J Emerg Med. 2011;29(6):613-617.

10. Lin BW, Yoshida D, Quinn J, Strehlow M. A better way to estimate adult patients' weights. Am J Emerg Med. 2009;27(9):1060-1064.

11. Crandall CS, Gardner S, Braude DA. Estimation of total body weight in obese patients. Air Med J. 2009;28(3):139-145.

12. Fawzy IA, Kamal NN. Stature and body weight estimation from various footprint measurements among Egyptian population. J Forensic Sci. 2010;55(4):884-888.

13. Miyatake N, Matsumoto S, Miyachi M, Fujii M, Numata T. Relationship between changes in body weight and waist circumference in Japanese. Environ Health Prev Med. 2007;12(5):220-223.

14. Donini LM, de Felice MR, de Bernardini L, et al. Body weight estimation in the Italian elderly. J Nutr Health Aging. 1998;2(2):92-95.

15. Bernal-Orozco MF, Vizmanos B, Hunot C, et al. Equation to estimate body weight in elderly Mexican women using anthropometric measurements. Nutr Hosp. 2010;25(4):648-655.

16. Chumlea WC, Guo S, Roche AF, Steinbaugh ML. Prediction of body weight for the nonambulatory elderly from anthropometry. J Am Diet Assoc. 1988;88(5):564-568. 
17. Chittawatanarat K, Pruenglampoo S, Kongsawasdi S, et al. The variations of body mass index and body fat in adult Thai people across the age spectrum measured by bioelectrical impedance analysis. Clin Interv Aging. 2011;6:285-294.

18. Jung MY, Chan MS, Chow VS, et al. Estimating geriatric patient's body weight using the knee height caliper and mid-arm circumference in Hong Kong Chinese. Asia Pac J Clin Nutr. 2004;13(3):261-264.

19. Kalichman L, Kobyliansky E. Sex- and age-related variations of the somatotype in a Chuvasha population. Homo. 2006;57(2):151-162.

20. Heath BH, Carter JE. A modified somatotype method. Am J Phys Anthropol. 1967;27(1):57-74.

21. Koleva M, Nacheva A, Boev M. Somatotype, nutrition, and obesity. Rev Environ Health. 2000;15(4):389-398.
22. Gallagher D, Heymsfield SB, Heo M, Jebb SA, Murgatroyd PR, Sakamoto Y. Healthy percentage body fat ranges: an approach for developing guidelines based on body mass index. Am J Clin Nutr. 2000;72(3):694-701

23. Bloomfield R, Steel E, MacLennan G, Noble DW. Accuracy of weight and height estimation in an intensive care unit: Implications for clinical practice and research. Crit Care Med. 2006;34(8):2153-2157.

24. Bedogni G, Borghi A, Battistini N. Body water distribution and disease. Acta Diabetol. 2003;40(Suppl 1):S200-S202.

25. Kotler DP, Burastero S, Wang J, Pierson RN Jr. Prediction of body cell mass, fat-free mass, and total body water with bioelectrical impedance analysis: effects of race, sex, and disease. Am J Clin Nutr. 1996; 64(3 Suppl):489S-497S.

\section{Publish your work in this journal}

The International Journal of General Medicine is an international, peer-reviewed open-access journal that focuses on general and internal medicine, pathogenesis, epidemiology, diagnosis, monitoring and treatment protocols. The journal is characterized by the rapid reporting of reviews, original research and clinical studies across all disease areas.
A key focus is the elucidation of disease processes and management protocols resulting in improved outcomes for the patient.The manuscript management system is completely online and includes a very quick and fair peer-review system. Visit http://www.dovepress.com/ testimonials.php to read real quotes from published authors.

Submit your manuscript here: http://www.dovepress.com/international-journal-of-general-medicine-journal 